Hartman, P. E., Loper, J. C. \& Šerman, D. (1960). J. gen. Microbiol. 22, 323-353

\title{
Fine Structure Mapping by Complete Transduction Between Histidine-requiring Salmonella Mutants
}

\author{
By P. E. HARTMAN, J. C. LOPER and D. ŚERMAN \\ Department of Biology, The Johns Hopkins University, Baltimore 18, \\ Maryland, U.S.A.
}

\begin{abstract}
SUMMARY: The growth characteristics, accumulations and genetic tests carried out by means of complete transduction for over 200 independently isolated histidinerequiring (his) mutants of Salmonella typhimurium are described. Sites of mutation engendering the histidine-requiring phenotype all lie within a short chromosomal region. This region is divisible into seven smaller regions, or gene loci, each correlated with a specific physiological phenotype. The gene loci appear to be linearly arranged and are linked in the order $E, F, A, B, C, D$ and $G$. The five central loci are linked in an order corresponding to the sequence of reactions in the biosynthetic pathway for histidine. The functions of the two terminal loci, $E$ and $G$, are unknown. The loci are nearly, or truly adjacent. A few of the sites of mutation are accurately mapped within a single gene. All the evidence is consistent with the view that processes involved in intra- and inter-genic recombination are identical. Some characteristics of multisite mutations are described. Several multisite mutations are interpreted as due to deletion of genetic material while one (his-57) is interpreted as due to an inversion, with or without concomitant deletion of an adjacent region. Single-site and multisite mutations exert specific effects on recombination frequencies in nearby regions.
\end{abstract}

Data previously obtained from analyses of 33 histidine-requiring mutants of Salmonella typhimurium (Hartman, 1956) indicated that at least four closely linked genes were involved in as many different steps of histidine synthesis. Some evidence was presented which indicated that the genes were linked in an order corresponding to the sequence of reactions they controlled in the biosynthetic pathway. This order was derived from a comparison of genetic mapping, by means of the frequency with which two his mutants recombine to produce prototrophs, with studies on precursor accumulations and growth responses. The mutations were correlated with genetic blocks in a pathway of biosynthesis analogous to that previously described for Neurospora (Ames, Garry \& Herzenberg, 1960). Further analysis has extended the basic findings of the initial report with regard to the fine structure of the histidine region of the Salmonella chromosome. Data obtained in growth and in recombination tests will be presented in this paper. The companion papers describe the functional behaviour of the genetic units as detected by abortive transduction (Hartman, Hartman \& Serman, 1960) and on the enzyme content of some of the mutants (Ames et al. 1960).

\section{METHODS}

Nomenclature. The system of nomenclature for the designation of bacterial mutants follows the scheme outlined by Demerec (1956a) as recently modified (Nomenclature, 1958). The following terms will be used in this and the 
companion paper: (1) gene, a discrete section of the chromosome governing the presence of a specific protein molecule(s), for example an enzyme, probably through the determination of a single protein species; (2) locus, or gene locus, the place on the chromosome occupied by a gene in any of its forms, wildtype or mutant; (3) allele, an entire gene, regardless of its exact configuration (wild-type or any one of a number of mutant forms); (4) site, a region of a gene, altered in the mutant, which can recombine with adjacent sites; (5) singlesite mutation, a mutation which has so far been detected as involving only a single site of a single gene; (6) set, two or more mutants or mutant alleles, identical or non-identical, altered in the same single site; (7) multisite mutation, a mutation which demonstrably involves two or more adjacent sites of either a single gene or of adjacent genes; (8) complementation unit, a functional unit, extending over several or many adjacent sites, and defined by the inability of all of its recessive alleles to complement all other recessive alleles in complementation tests within the unit.

Bacterial strains. Table 1 lists the derivations of the mutant strains of Salmonella typhimurium used in this study. Other multiple-auxotrophic mutants were described elsewhere (Hartman, 1956). Following the isolation of histidine-requiring mutants from auxotrophic parental strains, the auxotrophic markers of the parental strains were transduced to wild-type with H-4 phage, and non-lysogenic sensitive clones obtained (Hartman, 1956). Since the rates of spontaneous mutation in strain LT-2, and, especially, in LT-7 are quite high, it is probable that the majority of mutants derived from pretreated cultures were already present as spontaneous mutants. This is true in all cases except with 2-aminopurine, a potent mutagen for Salmonella (Dr M. Demerec, personal communication). Back-mutation tests with mutants obtained with 2-aminopurine treatment indicate that many of them have characteristics different from spontaneous mutants (Dr C. Kirchner, personal communication). Selection of all mutants was performed by the penicillin screening method (Lederberg, 1950), in most cases with 50 units penicillin/ ml. culture. The organisms from saturated aerated nutrient broth cultures of the parental strains were washed with saline and suspended in the penicillincontaining medium at $c .5 \times 10^{8}$ bacteria $/ \mathrm{ml}$. for the isolation of mutants from strain LT-2, or at $c .1 \times 10^{8} \mathrm{bacteria} / \mathrm{ml}$. for strain LT-7. After incubation for 18-24 $\mathrm{hr}$. without aeration, 0.1 and $0.01 \mathrm{ml}$. samples were plated on the enriched minimal agar described below. Saline suspensions of presumptive mutant (small) colony organisms were streaked on minimal agar and agar supplemented with various pools of growth factors for detection of auxotrophs and purification as single clones, or were streaked on nutrient agar and replicaplated (Lederberg \& Lederberg, 1952) the following day on supplemented media. The growth-factor pools were arranged such that each single factor appeared in two separate pools, enabling immediate identification in most cases of the specific supplement required by the mutant (Kaudewitz, Vielmetter \& Friedrich-Freksa, 1958).

Bacterial stocks were kept at room temperature (about $24^{\circ}$ ) as stab cultures in cork-stoppered, paraffin-sealed vials containing $0.9 \%(w / v)$ dehydrated 
Difco-nutrient broth, $0.75 \%(\mathrm{w} / \mathrm{v})$ agar, and $0.5 \%(\mathrm{w} / \mathrm{v})$ sodium chloride. Working stocks were maintained on agar slopes containing $1.5 \%$ (w/v) dehydrated Difco-nutrient broth, $2.5 \%(\mathrm{w} / \mathrm{v})$ agar and $0.5 \%(\mathrm{w} / \mathrm{v})$ sodium chloride and were retained in the refrigerator at $c .4^{\circ}$.

Phage stocks. PLT22 phage (P22) suspensions were prepared, assayed, and stored as detailed previously (Hartman, 1956).

\section{Table 1. Sources of mono-auxotrophic histidine-requiring mutants of Salmonella typhimurium and methods utilized in their isolation}

The parental strains were Salmonella typhimurium strains LT-2 and LT-7 or their derivatives. Most mutants were isolated from untreated nutrient broth-grown cultures (Spont.). Several were isolated after ultraviolet, X-ray, or lysozyme treatment and subsequent growth in broth or were grown in broth containing 2-aminopurine (see text). Monoauxotrophic derivatives were obtained from poly-auxotrophs by complete transduction and purification of $\mathbf{P 2 2}$-sensitive clones (transd.). The numbers in the third column indicate the isolation and permanent stock numbers of the histidine-requiring mutants.

\begin{tabular}{|c|c|c|}
\hline Parental strain & Method & Mutants \\
\hline \multicolumn{3}{|l|}{ LT-2 } \\
\hline Wild-type & Spont. & $12,13,18,39-61,89,162,163,453$ \\
\hline Wild-type & Ultraviolet & $1-9,62,195-199$ \\
\hline Wild-type & X-rays & $53,54,195-199$ \\
\hline Wild-type & 2-Aminopurine & $432-452$ \\
\hline Wild-type & Lysozyme & 63 \\
\hline ara -7 & Spont. & 189,190 \\
\hline ara-9 & Spont. & $183-188$ \\
\hline$a r a-9$ & 2-Aminopurine & $410-431$ \\
\hline ath-2 & Ultraviolet, transd. & $34-37$ \\
\hline cys-12 & Ultraviolet, transd. & $11,14,15$ \\
\hline cys -16 & Spont., transd. & $20,21,24$ \\
\hline cys $-12+$ try -8 & Spont., transd. & 27 \\
\hline cys -12 try -8 & Spont., transd. & 22 \\
\hline his-2 & Spont. & 211 \\
\hline pro- $21^{+}$ & Spont. & $102,103,129-152$ \\
\hline $\operatorname{try}-2$ & Spont., transd. & 31,33 \\
\hline \multicolumn{3}{|l|}{ LT-7 } \\
\hline Wild-type & Spont. & $64-88,90-95,212-263$ \\
\hline Wild-type, with mutator & Spont. & $169-182,303-305$ \\
\hline Wild-type, without mutator & Spont. & $164-168$ \\
\hline gal-24 & Ultraviolet & 10 \\
\hline gal-24 try-7 & Spont., transd. & $28-30,153,161$ \\
\hline pro- 47 & Spont., transd. & 401,402 \\
\hline pro- $47^{+}$ & Spont. & $\begin{array}{l}96-101,104-127,191-194,264-302 \text {, } \\
400\end{array}$ \\
\hline pro-51 str-r fer & Spont., transd. & $403-407$ \\
\hline
\end{tabular}

Media. Difco-nutrient broth was used whenever complex media was desired. Two minimal media were used: (1) the minimal medium of the Cold Spring Harbor Laboratory (see Hartman, 1956); (2) the ' $E$ ' medium of Vogel \& Bonner (1956). Enriched minimal medium (EM medium) consisted of one of the above to which $1.25 \%(v / v)$ liquid Difco-nutrient broth had been added. The choice of enrichment is important since some complex digests allow background residual growth of histidine mutants which severely inhibits the appearance of wild-type colonies (Dr B. A. D. Stocker, personal 
communication). Only very slight inhibition is noted when L-histidine or nutrient broth is used for enrichment.

Growth studies. Growth responses on solid medium were performed as previously indicated (Hartman, 1956). For measurement of growth in aerated liquid $\mathbf{E}$ medium at $37^{\circ}$, the optical density of cultures was followed turbidimetrically at $540 \mathrm{~m} \mu$ with a Klett-Summerson photoelectric colorimeter. In most cases the starting culture consisted of approximately $1 \times 10^{8}$ bacteria $/ \mathrm{ml}$. in order that the entire growth curve could be followed.

Accumulations. Minimal medium, containing L-histidine (free base; $2 \mu \mathrm{g} . /$ ml.) and D-histidine $\mathrm{HCl}(6 \mu \mathrm{g} . / \mathrm{ml}$.) was inoculated with $1 \%(\mathrm{v} / \mathrm{v})$ of a saturated nutrient broth aerated culture of the mutant strain. Glucose (0.01 $\mathrm{M})$ or glycerol $(0.02 \mathrm{M})$ served as effective major carbon sources, although some citrate was also present in the minimal medium. The cultures were aerated at $37^{\circ}$, preferably by shaking, for $48 \mathrm{hr}$. or longer. At $48 \mathrm{hr}$. all of the histidine in the medium had been used. Following plating to test for the presence of prototrophs, the cultures were centrifuged and the supernatant fluids chromatographed.

A colour reaction in test tubes (Bratton \& Marshall, 1939) was used to detect diazotizable amines; readings were performed at $540 \mathrm{~m} \mu$, close to the peak of adsorption for the predominant diazotizable amine accumulated by the Salmonella mutants.

The chromatographs were sprayed according to the modified Pauly and the copper sulphate techniques of Ames \& Mitchell (1952, 1955). In some cases, the paper chromatogram Bratton-Marshall procedure of Gots (Hartman, 1956) was also used for detecting diazotizable amines. Comparisons were made on the same chromatograms between various supernatant fluids and between mixtures of some supernatant fluids and known compounds (see below). The two solvent systems of Ames \& Mitchell (1952) were used. Compounds were tentatively identified when they had the same $R_{f}$ values, the same appearance under ultraviolet radiation and the same hue following the spray tests, as did the known compounds. Compounds which were presumptively imidazoleglycerol phosphate ester and L-histidinol phosphate ester were isolated free from other Pauly-positive substances, partially hydrolysed, and the free bases identified by chromatography.

Chemicals. Some of the compounds used in this work were listed with their sources in Hartman (1956). Other chemicals received through the courtesy of $\mathrm{Dr} \mathrm{B}$. N. Ames were: L-histidinol.2HCl; $\mathrm{N}$ - $\alpha$-formyl-L-histidine; histidylleucine; spinacin; imidazole-benzyl-L-histidine; D-arabino-tetrahydroxybutylimidazole; 3-pyrazole alanine; 2-thiazole alanine; 4-thiazole alanine; 3amino-1: 2:4-triazole; 2-imidazole alanine, 2-thiazole alanine. Other chemicals (purchased from California Corporation for Biochemical Research, Los Angeles 63, California, U.S.A.) were: $\mathrm{N}$-acetyl-L-histidine. $\mathrm{H}_{2} \mathrm{O}$; L-histidine methyl ester. $\mathrm{HCl}$; histamine. $2 \mathrm{HCl}$; 4-amino-5-imidazole-carboxamide. $\mathrm{H}_{2} \mathrm{O}$; 3-methyl histidine. A sample of histidylhistidine was purchased from Mann Research Labs, Inc., New York 6, New York. L-Histidinol and L-histidinol phosphate ester were isolated from the supernatant fluids of Salmonella typhimurium 
mutant his-141 grown in minimal medium with limiting histidine. Imidazole glycerol and imidazole glycerol phosphate ester were synthesized and isolated according to a procedure modified after Ames (1957). $N$ - $\alpha$-Formyl-L-histidine was synthesized by the procedure of Abderhalden \& Weil (1912).

In most of the experiments reported in this paper, L-histidine (free base) and D-histidine. $\mathrm{HCl}$ (from Nutritional Biochemicals Corporation, Cleveland 28, Ohio, U.S.A.) were used.

Transduction tests. Genetic tests were performed using P22-sensitive recipient Salmonella typhimurium and with EM as the plating medium (cf. Hartman, 1956). In most experiments, multiplicities were maintained at about 1 phage/bacterium. Bacteria were grown overnight in nutrient broth at $37^{\circ}$ with forced aeration $\left(2-4 \times 10^{9}\right.$ colony-forming units $/ \mathrm{ml}$.). Bacteria were sedimented at room temperature by centrifugation at $3,500 \times g$. for $10 \mathrm{~min}$. from this saturated culture and resuspended and diluted 1/5 in T2 buffer (Hershey \& Chase, 1952) or in single-strength $\mathbf{E}$ medium (without carbon source). Fresh cultures were used in all of the tests reported in this paper; however, bacteria suspended in $\mathrm{T} 2$ buffer and retained at $4^{\circ}$ maintain completely for a period of 3 weeks their viability and efficiency as recipients in transduction tests. Samples of the bacterial suspension were placed in Wassermann tubes and phage added. Following incubation for $5 \mathrm{~min}$. at $37^{\circ}$ to allow absorption, $0.1 \mathrm{ml}$. samples were spread evenly on agar Petri plates. Control platings consisted of bacteria to which homologous phage and to which T2 buffer alone were added.

\section{RESULTS}

\section{Growth responses}

All mutants listed in Table 1 grew maximally on agar medium supplemented with L-histidine (base $20 \mu \mathrm{g} . / \mathrm{ml}$.) and failed to grow, or grew poorly, on medium lacking this supplement. Mutants which exhibited slow growth at $37^{\circ}$ in the absence of histidine are termed 'leaky' mutants. Leaky mutants are: his-6, -32, -56, -59, -62, -76, -111, -116, -119, -140, -141, -143, -145, -163, -166 $-168,-174,-176,-177,-196,-211,-214,-222,-270,-273,-278,-280$. Many of the leaky mutants were stimulated by the addition to the medium of any of a number of purine bases (Hartman, 1956) or adenosine or guanosine. Some other strains, not observed to be leaky by the crude tests used, were also stimulated by purines: $h i s-48,-58,-80,-81,-106,-122,-144,-147,-169,-212$, $-248,-253,-255$. Several strains exhibited heavier growth on minimal medium at $24^{\circ}$ than at $37^{\circ}$; these temperature-sensitive strains were: $h i s-59,-107,-211$, and -232 . One strain, his-76, grew better on minimal medium at $37^{\circ}$ than at $24^{\circ}$. The above responses are comparatively homogeneous responses of the majority of the bacteria in the populations.

Table 2 summarizes a number of attributes, including growth responses, of the major classes of the histidine mutants used. When nutrient broth cultures were placed directly on minimal medium, a few divisions took place with mutants of groups $F, A, H$, and $G$, resulting in the formation of 
heterogeneously sized round microcolonies. $E$ mutants formed heterogeneously sized, 'snaky'-appearing microcolonies. Not more than one or two divisions were engendered from $B, C$ and $D$ mutants, except for the relatively homogeneously sized microcolonies formed by mutants which carried leaky alleles of these genes. On enriched minimal agar plates, mutants designated as group $\boldsymbol{E}$ mutants uniquely showed a heavy residual growth consisting microscopically of elongate bacteria in eccentric microcolonies. Mutant his-E, $F-135$ exhibited the behaviour of $F$ mutants in the above two respects.

In liquid minimal medium with glucose or glycerol as carbon source, wildtype strain LT-2 grew with a generation time of $c .50 \mathrm{~min}$ at $37^{\circ}$ and reached a

Table 2. Summary of the properties of the seven general phenotypic classes of his mutants of Salmonella typhimurium

\begin{tabular}{|c|c|c|c|c|c|c|c|c|c|c|c|c|}
\hline \multirow[b]{2}{*}{ Class } & \multirow{2}{*}{$\begin{array}{c}\text { Residual } \\
\text { growth on } \\
\text { minimal } \\
\text { medium }\end{array}$} & \multirow{2}{*}{$\begin{array}{c}\text { Residual } \\
\text { growth on } \\
\text { enriched } \\
\text { minimal } \\
\text { medium }\end{array}$} & \multirow{2}{*}{$\begin{array}{c}\text { Growth on } \\
\text { L-histi- } \\
\text { dinol }\end{array}$} & \multirow{2}{*}{$\begin{array}{c}\text { Major } \\
\text { accumu- } \\
\text { lations* }\end{array}$} & \multicolumn{6}{|c|}{ Enzyme content $\dagger$} & \multirow{2}{*}{$\begin{array}{l}\text { Responses } \\
\text { in } \\
\text { intergenic } \\
\text { comple- } \\
\text { mentation } \dagger\end{array}$} & \multirow{2}{*}{$\begin{array}{l}\text { Shape of } \\
\text { abortive } \\
\text { colonies } \dagger\end{array}$} \\
\hline & & & & & $\mathbf{F}$ & $\mathbf{A}$ & $\mathbf{B}$ & C & $\mathbf{P}$ & $\mathbf{D}$ & & \\
\hline $\boldsymbol{E}$ & $\underset{\text { (snaky) }}{+}$ & $+t$ & + & $\mathbf{0}$ & + & - & + & + & + & + & +++ & $\begin{array}{l}\text { Snaky, } \\
\text { oval }\end{array}$ \\
\hline $\boldsymbol{F}$ & + & + & + & $\mathbf{0}$ & $\mathbf{0}$ & + & + & + & + & + & + & Oval \\
\hline $\boldsymbol{A}, \boldsymbol{H}$ & + & + & + & $\mathbf{0}$ & + & $\mathbf{0}$ & + & + & + & $t$ & + & Oval \\
\hline $\boldsymbol{B}$ & o & + & + & 1,2 & - & . & 0 & + & + & + & + & Oval \\
\hline $\boldsymbol{C}$ & $\mathbf{0}$ & + & + & $1,2,3,4,5$ & . & . & + & $\mathbf{0}$ & + & + & + & Oval \\
\hline $\boldsymbol{D}$ & $\mathbf{0}$ & + & o & $\begin{array}{c}1,2,3,4,5 \\
6,7\end{array}$ & - & . & + & + & + & $\mathbf{0}$ & + & Oval \\
\hline $\boldsymbol{G}$ & + & + & $++t$ & 0 & - & $\cdot$ & + & + & + & + & + & Oval \\
\hline+ & $+t+$ & +++ & - & 0 & + & + & + & + & + & + & + & Oval \\
\hline
\end{tabular}

* Abbreviations : (1) imidazoleglycerol phosphate ester; (2) imidazole glycerol; (3) imidazoleacetol phosphate ester; (4) imidazoleacetol; (5) imidazole lactic acid; (6) histidinol phosphate ester; (7) histidinol.

$\dagger$ Abbreviations: (F) synthesis of compound III; (A) conversion of compound III to imidazoleglycerol phosphate ester; (B) imidazole-glycerol phosphate ester dehydrase; (C) imidazoleacetol phosphate ester transaminase; (P) L-histidinol phosphate ester phosphatase; (D) L-histidinol dehydrogenase. Information on enzyme content is extended to classes of mutants from assays of but one, or a few, members of each phenotypic class. $\mathbf{A}(+)$ indicates that the enzyme activity was present in cell-free extracts and $(0)$ indicates that the enzyme activity was looked for but not detected. Brief mention of activities for enzymes F and A are from Moyed \& Magasanik (1957); and Magasanik (1958) (personal communication); quantitative data are available for enzymes B, C, P, and D in Ames et al. (1960) and Ames (personal communication).

+ Refer to Hartman et al. (1960).

plateau at about $4 \times 10^{9}$ bacteria/ml. The addition of L-histidine (free base $20 \mu \mathrm{g} . / \mathrm{ml}$.) did not influence the generation time nor the plateau at culture saturation as determined turbidimetrically, by bacterial counts in a PetroffHausser chamber, or by plate counts. The same generation time and position of the plateau in stationary phase was also characteristic of all mutant strains examined on L-histidine (20 $\mu \mathrm{g} . / \mathrm{ml}.):$ his $-3,-6,-8,-9,11,-12,-13,-22,-52,-53$, $-57,-63,-79,-80,-105,-135,-145,-148,-150,-152$. On limiting concentrations of L-histidine, the growth rates of mutant strains equalled that of wild-type bacteria but then abruptly stopped (for most mutants) or changed to a slower logarithmic growth phase (for leaky mutants and with $E$ mutants). This behaviour is shown in Fig. 1 for hisE-11 at two L-histidine concentrations. The 
second logarithmic growth phase was not always so striking even though attempts were made to keep all conditions standard or to enhance this phase by altering the growth medium. A growth curve for the wild-type on minimal medium is included for comparison. Growth curves of wild-type in the presence of 2,4 or $20 \mu \mathrm{g}$. L-histidine/ml. and of his-11 with $20 \mu \mathrm{g}$. L-histidine/ml. were very similar to that shown for the wild-type on minimal medium. Addition of adenine $(20 \mu \mathrm{g} . / \mathrm{ml}$.$) and thiamine (4 \mu \mathrm{g} . / \mathrm{ml}$.) neither appreciably spared histidine nor changed the growth rates of hisE-11 grown on limiting histidine.

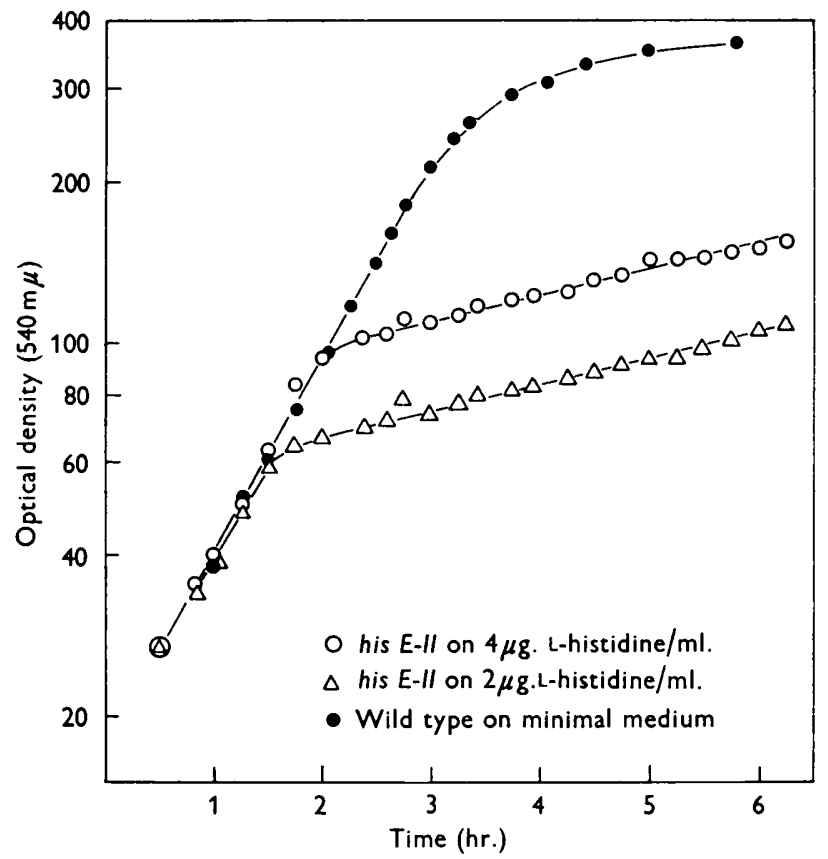

Fig. 1. Growth of Salmonella typhimurium mutant his-E11 on limiting histidine and wild-type (LT-2) on minimal medium.

The addition of L-histidinol. $2 \mathrm{HCl}(30 \mu \mathrm{g} . / \mathrm{ml}$.) to minimal agar stimulated the growth of some mutants but not others. The results for individual mutants are given in table 3 and are summarized in Table 2 with regard to classes of mutants. Histidinol failed to stimulate the growth of all mutants tested involving gene $D$, either by mutation within gene $D$ (Figs. 3, 5) or by 'position effect' (Hartman et al. 1960). The only exception was hisD-111, a leaky mutant. All other mutants tested grew on L-histidinol; the growth of $G$ mutants and a few leaky mutants (e.g. hisC-62, hisB-116) was much more rapid than that of other mutants. The rate of growth was nevertheless low compared with the rate on L-histidine. The growth responses of almost all mutants were heterogeneous, papillae forming when the bacteria were spread on histidinol-containing agar (compare Vogel, Davis \& Mingioli, 1951). Some clones retained their ability for rapid growth on histidinol after several transfers in complete medium (nutrient broth); most of them did not grow on minimal medium. 
Table 3. Some characteristics of his mutants of Salmonella typhimurium

Column I. Immediately after isolation and characterization of L-histidine as primary growth requirement, each mutant was given an italicized three-letter symbol, (his), denoting this requirement as well as a permanent isolation and stock number (Demerec, 1956a). Column II. The locus (or loci) in which the mutational change is localized, as determined by transductional and biochemical analyses, is designated by a capital letter (Demerec, $1956 a$ ). Where more than one functional unit is detected within the locus by complementation tests, the unit or units completely defective in the mutant are denoted by small letters (Hartman et al. 1960). Column III. Growth responses on agar containing L-histidinol $(20$ or $30 \mu \mathrm{g} / \mathrm{ml}$.) are recorded as negative (equivalent to minimal medium alone $=0$ ), slow growth with varying numbers of papillae $(+)$ and comparatively rapid growth $(+++)$.

Column IV. Accumulations: none $=$ no Pauly positive imidazoles detected; $0=$ diazotizable amine level similar to wild-type; 1 = diazotizable amine appreciably higher than wild-type; 2 = imidazoleglycerolphosphate ester; $3=$ imidazoleglycerol; $4=$ imidazoleacetol phosphate ester; $5=$ imidazoleacetol; $6=$ imidazole lactic acid; $7=1$-histidinol phosphate ester; $8=$ L-histidinol; $9=$ unidentified Pauly positive substance giving a pink reaction and having a high $\boldsymbol{R}_{f}$ value in propanol + acetic acid solvent.

\begin{tabular}{|c|c|c|c|c|c|c|c|}
\hline $\begin{array}{c}\text { Mutant } \\
\text { no. }\end{array}$ & $\begin{array}{c}\text { II } \\
\text { Locus } \\
\text { and } \\
\text { subunit }\end{array}$ & $\begin{array}{c}\text { III } \\
\text { Growth } \\
\text { on } \\
\text { his'ol }\end{array}$ & $\begin{array}{l}\text { Accumu- } \\
\text { lations }\end{array}$ & $\begin{array}{c}\text { Mutant } \\
\text { no. }\end{array}$ & $\begin{array}{c}\text { II } \\
\text { Locus } \\
\text { and } \\
\text { subunit }\end{array}$ & $\begin{array}{c}\text { III } \\
\text { Growth } \\
\text { on } \\
\text { his'ol }\end{array}$ & $\begin{array}{l}\text { Accumu- } \\
\text { lations }\end{array}$ \\
\hline his- 1 & $\mathbf{D b}$ & $\mathbf{0}$ & $3,6,7,8$ & his- 40 & Bd & + & $2, \mathbf{3}, \mathbf{9}$ \\
\hline-2 & $\mathbf{C}$ & + & $\underset{9}{1,2,3,4,5,6}$ & $\begin{array}{l}-41 \\
-42\end{array}$ & $\begin{array}{l}\mathbf{F} \\
\mathbf{F}\end{array}$ & $\begin{array}{l}+ \\
+\end{array}$ & $\begin{array}{l}0, \text { none } \\
0, \text { none }\end{array}$ \\
\hline-3 & $\mathbf{A}$ & + & 1 , none & -43 & C & + & $3,4,5,9$ \\
\hline - 6 & $\mathbf{F}$ & + & 1 , none & -44 & $\mathbf{F}$ & + & 0, none \\
\hline-8 & C & + & $\begin{array}{l}2,3,4,5,6 \\
9\end{array}$ & $\begin{array}{l}-45 \\
-46\end{array}$ & $\begin{array}{l}\mathbf{F} \\
\mathbf{G}\end{array}$ & $\begin{array}{c}+ \\
++t\end{array}$ & $\begin{array}{l}\text { 0, none } \\
\text { none }\end{array}$ \\
\hline-9 & Dab & $\mathbf{0}$ & $\begin{array}{c}3,4,5,6,7 \\
8\end{array}$ & $\begin{array}{l}-47 \\
-48\end{array}$ & $\begin{array}{l}\text { Bd } \\
\mathbf{A}\end{array}$ & $\begin{array}{l}+ \\
+\end{array}$ & $\begin{array}{l}2,3 \\
1, \text { none }\end{array}$ \\
\hline-10 & Da & $\mathbf{0}$ & $0,4,5,7,8$ & -49 & Db & $\mathbf{0}$ & $6,7,8$ \\
\hline-11 & Ebc & + & 1 , none & -50 & C & + & $4,5,6$ \\
\hline-12 & $\mathbf{B a}$ & + & $1,2,3$ & -52 & G & $+t+$ & None \\
\hline-13 & C & + & $\begin{array}{c}2,3,4,5,6 \\
9\end{array}$ & $\begin{array}{l}-53 \\
-54\end{array}$ & $\begin{array}{l}\text { Bd } \\
\text { Dab }\end{array}$ & $\stackrel{+}{0}$ & $\begin{array}{l}0,2,3 \\
4,5,8\end{array}$ \\
\hline-14 & $\mathbf{B a}$ & + & 2,3 & -55 & $\mathbf{F}, \mathbf{A}$ & + & 0, none \\
\hline-15 & C & + & $2,3,4,5,6$ & -56 & $\mathbf{B c}$ & + & 2,3 \\
\hline-18 & Dab & $\mathbf{0}$ & $6,7,8$ & -57 & E-G & $\mathbf{0}$ & None \\
\hline-20 & $\mathbf{B a}$ & + & 2,3 & -58 & $\mathbf{F}$ & $t$ & 1, none \\
\hline-21 & Babcd & + & 2,3 & -59 & Bc & + & 2,3 \\
\hline-22 & Babcd & + & $1,2,3$ & -60 & Dab & 0 & $4,5,6,8$ \\
\hline-23 & Da & $\mathbf{0}$ & $4,5,6,7,8$ & -61 & $\mathbf{B b}$ & + & 2,3 \\
\hline-24 & $\mathbf{B a}$ & + & $\mathbf{2}, \mathbf{3}, \mathbf{9}$ & -62 & C & $+t+$ & $3,4,5,6$ \\
\hline-27 & Dab & $\mathbf{0}$ & $6,7,8$ & -63 & $\mathbf{D}, \mathbf{G}$ & $\mathbf{0}$ & None \\
\hline-28 & C & + & $2,3,4,5,6$ & -64 & D & $\mathbf{0}$ & $0,7,8$ \\
\hline-29 & Babed & + & 2,3 & -65 & Bd & + & $2, \mathbf{3}, \mathbf{9}$ \\
\hline-30 & $\mathbf{A}$ & + & 1, none & -66 & Db & $\mathbf{0}$ & $6,7,8$ \\
\hline-31 & C & + & $2, \mathbf{3}, \mathbf{4}, \mathbf{5}, \mathbf{6}$ & -67 & $\mathbf{A}$ & + & o, none \\
\hline-32 & $\mathbf{H}$ & + & 1 , none & -68 & Db & $\mathbf{0}$ & $6,7,8,9$ \\
\hline-33 & $\mathbf{A}$ & + & 1, none & -69 & $\mathbf{A}$ & + & 0, none \\
\hline-34 & Dab & 0 & $\begin{array}{l}4,5,6,7,8 \\
9\end{array}$ & $\begin{array}{r}-70 \\
-71\end{array}$ & $\begin{array}{l}\mathbf{G} \\
\mathbf{F}\end{array}$ & $\begin{array}{l}+ \\
+\end{array}$ & $\begin{array}{l}\text { None } \\
0, \text { none }\end{array}$ \\
\hline-35 & Ec & + & 1, none & -72 & $\mathbf{G}$ & +++ & None \\
\hline-36 & $\mathbf{D b}$ & $\mathbf{0}$ & $3,4,5,6,7$, & $\begin{array}{l}-72 \\
-74\end{array}$ & $\begin{array}{l}\text { F } \\
\mathbf{D}\end{array}$ & $\begin{array}{l}+ \\
+\end{array}$ & $\begin{array}{l}0, \text { none } \\
6,8\end{array}$ \\
\hline-37 & Db & 0 & $\begin{array}{l}3,4,5,6,7 \\
8\end{array}$ & $\begin{array}{l}-75 \\
-76\end{array}$ & $\mathbf{F}$ & $\begin{array}{l}+ \\
+\end{array}$ & $\begin{array}{l}0, \text { none } \\
0, \text { none }\end{array}$ \\
\hline $\begin{array}{l}-38 \\
-39\end{array}$ & $\begin{array}{l}\mathbf{A} \\
\mathbf{D} \mathbf{b}\end{array}$ & $\begin{array}{l}+ \\
0\end{array}$ & $\begin{array}{l}1, \text { none } \\
3,5,7,8\end{array}$ & $\begin{array}{l}-77 \\
-78\end{array}$ & $\begin{array}{l}\text { Db } \\
\text { Dab }\end{array}$ & $\begin{array}{l}\mathbf{0} \\
\mathbf{0}\end{array}$ & $\begin{array}{l}5,6,7,8,8 \\
5,6,8\end{array}$ \\
\hline
\end{tabular}


Table 3 (cont.)

\begin{tabular}{|c|c|c|c|c|c|c|c|}
\hline $\begin{array}{c}\text { I } \\
\text { Mutant } \\
\text { no. }\end{array}$ & $\begin{array}{l}\text { II } \\
\text { Locus } \\
\text { and } \\
\text { subunit }\end{array}$ & $\begin{array}{l}\text { III } \\
\text { Growth } \\
\text { on } \\
\text { his'ol }\end{array}$ & $\begin{array}{c}\text { IV } \\
\text { Accumu- } \\
\text { lations }\end{array}$ & $\begin{array}{c}\text { Mutant } \\
\text { no. }\end{array}$ & $\begin{array}{c}\text { II } \\
\text { Locus } \\
\text { and } \\
\text { subunit }\end{array}$ & $\begin{array}{l}\text { III } \\
\text { Growth } \\
\text { on } \\
\text { his'ol }\end{array}$ & $\begin{array}{c}\text { IV } \\
\text { Accumu- } \\
\text { lations }\end{array}$ \\
\hline his- 79 & $\mathbf{B a}$ & + & $2,3,9$ & his-135 & Eabed, F & + & 0 , none \\
\hline-80 & A & + & 1 , none & -136 & Bc & + & \\
\hline-81 & A & + & 1 , none & -137 & D & 0 & $0.5,6,7,8$ \\
\hline-82 & Db & $\mathbf{0}$ & $\begin{array}{c}2,3,4,5,6 \\
8,9\end{array}$ & $\begin{array}{l}-138 \\
-139\end{array}$ & $\begin{array}{l}\text { Babcd } \\
\text { Dab }\end{array}$ & $\begin{array}{l}+ \\
0\end{array}$ & $\begin{array}{l}2,3 \\
0,7,8\end{array}$ \\
\hline-83 & Db & 0 & $4,5,6,8,9$ & -140 & A & + & 1 , none \\
\hline-84 & $\mathbf{D b}$ & o & $4,5,8,9$ & -141 & $\mathrm{Db}$ & 0 & $0,7,8$ \\
\hline-85 & Dab & $\mathbf{0}$ & $3,5,6,8$ & -142 & Dab & 0 & $0,6,8$ \\
\hline-86 & $\mathbf{C}$ & + & $4,5,6$ & -143 & Bd & + & 2,3 \\
\hline-87 & C & + & $0,5,6$ & -144 & $\mathbf{F}$ & + & 1 , none \\
\hline-88 & D & $\mathbf{0}$ & $0,7,8$ & -145 & Eabcd & + & 0, none \\
\hline-89 & $\mathbf{A}$ & + & 0 , none & -146 & C & + & $3,4,6$ \\
\hline-90 & $\mathbf{D b}$ & o & $5,6,8$ & -147 & $\mathbf{F}$ & + & 1 , none \\
\hline-91 & F & + & 0 , none & -148 & F & + & 0, none \\
\hline-92 & Db & $\mathbf{0}$ & $6,7,8$ & -149 & Dab & o & $0,6,7,8$ \\
\hline-93 & F & + & 0 , none & -150 & D & $\mathbf{0}$ & $0,7,8$ \\
\hline-94 & $\mathbf{D}$ & 0 & $2,3,6,8$ & -151 & C & + & $3,4,5,6$ \\
\hline-95 & $\mathbf{F}$ & + & 0 , none & -152 & F-D & o & 0 , none \\
\hline-96 & & ++ & 0, none & -153 & $\mathbf{A}$ & + & None \\
\hline-97 & A & + & 1, none & -154 & Dab & $\mathbf{0}$ & $4,6,8$ \\
\hline-99 & $\mathbf{E a}$ & + & 0 , none & -155 & F & + & None \\
\hline-100 & $\mathbf{A}$ & + & 1, none & -156 & • & $+t$ & None \\
\hline-101 & & +++ & 0, none & -157 & $\mathbf{A}$ & + & None \\
\hline-102 & Bed & ++ & 3 & -158 & F & + & None \\
\hline-103 & $\mathbf{F}$ & + & 0 , none & -159 & $\mathbf{A}$ & . & None \\
\hline-104 & C & + & $4,5,6$ & -160 & D & 0 & 7,8 \\
\hline-105 & Ed & + & 0 , none & -161 & & + & $3,5,6.9$ \\
\hline-106 & $\mathbf{A}$ & + & 1 , none & -162 & $\mathbf{E}$ & + & None \\
\hline-107 & $\mathbf{H}$ & + & 1, none & -163 & C & + & $4,5,6,9$ \\
\hline-108 & Db & 0 & $6,7,8$ & -164 & $\mathbf{E}$ & + & 1 , none \\
\hline-109 & $\mathbf{F}$ & + & 1 , none & -165 & & + & 0, none \\
\hline-110 & C & + & $3,4,5,6$ & -166 & D & 0 & $1,7,8$ \\
\hline-111 & $\mathrm{Db}$ & + & 7,8 & -167 & Bc & + & $\mathbf{2}, \mathbf{3}$ \\
\hline-112 & Da & 0 & $4,5,8$ & -168 & & + & 1, none \\
\hline-113 & $\mathrm{Db}$ & o & $3,4,5,8,9$ & -169 & $\mathbf{c}$ & + & 4,5 \\
\hline-114 & F & ++ & 0 , none & -170 & D & o & $0,6,7,8,9$ \\
\hline-115 & C & + & $3,4,5,6$ & -171 & D & o & $0,7,8.9$ \\
\hline-116 & Bc & ++ & 2,3 & -172 & & + & None \\
\hline-117 & C & + & $3,4,5,6$ & -173 & D & 0 & $0,7,8$ \\
\hline-118 & Babc & + & 2,3 & -174 & • & . & 0 , none \\
\hline-119 & G & ++ & none & -175 & 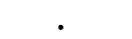 & . & o, none \\
\hline-120 & C & + & $3,4,5,6,9$ & -176 & D & o & $6,7,8$ \\
\hline-121 & C & + & $3,4,5,6$ & -177 & & + & 1 , none \\
\hline-122 & C & + & $1,4,5,6$ & -178 & D & o & None \\
\hline-123 & Dab & o & 7,8 & -179 & $\mathbf{A}$ & + & None \\
\hline-124 & Dab & $\mathbf{0}$ & $7,8,9$ & -180 & D & 0 & o, none \\
\hline-125 & $\mathbf{E a}$ & + & 0 , none & -181 & & $\cdot$ & o, none \\
\hline-126 & D & o & $5,7,8,9$ & -182 & $\mathbf{D}$ & $\mathbf{0}$ & $0,6,7,8$ \\
\hline-127 & Dab & $\mathbf{0}$ & $1,5,7,8,9$ & -195 & Babed & + & $0,2,3$ \\
\hline-129 & C & + & $4,5,6,9$ & -196 & B & + & $0,2,3$ \\
\hline-130 & $\mathbf{D b}$ & $\mathbf{0}$ & $6,7,8$ & -197 & $\mathbf{F}$ & + & 0 , none \\
\hline-131 & $\mathbf{F}$ & + & 0 , none & -198 & F & + & 0 , none \\
\hline-132 & $\mathbf{F}$ & + & 0 , none & -199 & G & +++ & 0 , none \\
\hline-133 & Dab & 0 & $6,7,8$ & -200 & G & + & None \\
\hline \multirow[t]{2}{*}{-134} & $\mathbf{A}, \mathbf{H}$ & + & 0 , none & -201 & C & + & $3,4,5,6$ \\
\hline & & & & & & & $2 \mathrm{X}-2$ \\
\hline
\end{tabular}




\begin{tabular}{|c|c|c|c|c|c|c|c|}
\hline & & & Table & (cont.) & & & \\
\hline I & II & III & IV & $\mathbf{I}$ & II & III & IV \\
\hline $\begin{array}{c}\text { Mutant } \\
\text { no. }\end{array}$ & $\begin{array}{l}\text { Locus } \\
\text { and } \\
\text { subunit }\end{array}$ & $\begin{array}{c}\text { Growth } \\
\text { on } \\
\text { his'ol }\end{array}$ & $\begin{array}{l}\text { Accumu- } \\
\text { lations }\end{array}$ & $\begin{array}{c}\text { Mutant } \\
\text { no. }\end{array}$ & $\begin{array}{l}\text { Locus } \\
\text { and } \\
\text { subunit }\end{array}$ & $\begin{array}{c}\text { Growth } \\
\text { on } \\
\text { his'ol }\end{array}$ & $\begin{array}{l}\text { Accumu- } \\
\text { lations }\end{array}$ \\
\hline his-202 & C & + & $4,5,6$ & his-251 & $\mathbf{F}$ & + & o, none \\
\hline-203 & $\mathbf{G}$ & $\mathbf{0}$ & 0, none & -252 & C & + & $3,4,5,6$ \\
\hline-204 & G & $t+t$ & None & -253 & $\mathbf{A}$ & + & 1 , none \\
\hline-205 & $\mathbf{G}$ & $+t+$ & None & -254 & Db & 0 & $0,6,7,9$ \\
\hline-206 & $\mathbf{B b}$ & + & $2,3,9$ & -255 & $\mathbf{G}$ & $+t+$ & None \\
\hline-207 & C & + & $3,4,5,6$ & -256 & $\mathbf{F}$ & + & 0 , none \\
\hline-208 & $\mathbf{D a}$ & $\mathbf{0}$ & $3,4,5,6,8,9$ & -257 & Bd & + & $0,2,3$ \\
\hline-209 & C & + & $3,4,6$ & -258 & $\mathbf{C}$ & + & $0,4,5,6$ \\
\hline-210 & C & + & $3,4,5,6$ & -259 & $\mathbf{E a}$ & + & 0 , none \\
\hline-211 & C & . & $3,4,5,6$ & -260 & $\mathbf{F}$ & + & 0, none \\
\hline-212 & C & + & 1, none & -261 & C & + & $4,5,6$ \\
\hline-213 & $\mathbf{B d}$ & + & 2,3 & -262 & Babcd & ++ & $0,2,3$ \\
\hline-214 & Db & 0 & $0,6,8$ & -263 & $\mathbf{A}$ & + & 0 , none \\
\hline-215 & D & 0 & $0,5,6,8$ & -264 & $\mathbf{E b}$ & . & 0, none \\
\hline-216 & D & 0 & $0,6,8$ & -265 & . & + & $0,4,5,6$ \\
\hline-217 & Bd & + & 2,3 & -266 & $\mathbf{E}$ & + & None \\
\hline-218 & $\mathbf{F}$ & + & 0, none & -267 & - & + & None \\
\hline-219 & $\mathbf{F}$ & + & 0, none & -268 & . & - & 1, none \\
\hline .220 & Dab & 0 & $0,6,8$ & -269 & $\mathbf{A}$ & . & 1 , none \\
\hline-221 & $\mathbf{F}$ & + & 0 , none & -270 & . & + & 0 , none \\
\hline-222 & $\mathbf{F}$ & + & 0 , none & -271 & D & o & $0,7,8$ \\
\hline-223 & D & $\mathbf{0}$ & $0,6,7,8$ & -272 & - & . & 0 , none \\
\hline-224 & $\mathbf{F}$ & + & 0 , none & -273 & D & o & $0,5,6,7,8,9$ \\
\hline-225 & $\mathbf{C}$ & + & $4,5,6$ & -274 & D & $\mathbf{0}$ & $0,6,7,8, \mathbf{9}$ \\
\hline-226 & Dab & $\mathbf{0}$ & $0,5,6,7,8$ & -276 & $\theta^{\circ}$ & + & 0, none \\
\hline-227 & $\mathbf{F}$ & + & o, none & -277 & $\mathbf{A}$ & . & o, none \\
\hline-228 & $\mathbf{F}$ & + & 0, none & -278 & . & + & 0,3 \\
\hline-229 & Bd & + & 2,3 & -280 & C & + & $0,4,5,6$ \\
\hline-230 & Dab & $\mathbf{0}$ & $0,5,6,8$ & -281 & . & . & 0 , none \\
\hline-231 & $\mathbf{A}$ & + & None & -282 & - & . & 0, none \\
\hline-232 & $\mathbf{F}$ & + & o, none & -283 & - & . & 0 , none \\
\hline-233 & Db & $\mathbf{0}$ & $5,6,8$ & -284 & $\mathbf{E b}$ & + & 1 , none \\
\hline-234 & Bbed & + & 2,3 & -285 & . & . & 0, none \\
\hline-235 & $\mathbf{F}$ & + & 0 , none & -286 & . & . & $0,2,3$ \\
\hline-236 & Db & o & $5,6,8$ & -287 & . & . & 1 , none \\
\hline-237 & $\mathrm{Db}$ & $\mathbf{0}$ & $0,5,6,8$ & -288 & . & . & 0, none \\
\hline-238 & Bd & + & 2,3 & -289 & Babcd & + & $1,2,3$ \\
\hline-239 & Db & $\mathbf{0}$ & $0,7,8$ & -290 & . & . & 1 , none \\
\hline-240 & $\mathbf{F}$ & + & 0, none & -291 & $\mathbf{E}$ & $\mathbf{0}$ & 0, none \\
\hline-241 & Bd & + & $0,2,3$ & -292 & $\mathbf{B}$ & + & $0,2,3$ \\
\hline-242 & $\mathbf{E a}$ & + & 0 , none & -293 & Bd & . & 0 , none \\
\hline-243 & Babc & + & 2,3 & -294 & $\mathbf{A}$ & + & 1 , none \\
\hline-244 & $\mathrm{Db}$ & $\mathbf{0}$ & $0,5,6,7,8$ & -295 & $\mathbf{D}$ & $\mathbf{0}$ & $1,7,8$ \\
\hline-245 & $\mathrm{Db}$ & $\mathbf{0}$ & $0,5,6,7,8$ & -296 & $\mathbf{F}$ & + & 0, none \\
\hline-246 & $\mathbf{A}$ & + & 1 , none & -298 & - & + & $0,5,6$ \\
\hline-247 & $\mathbf{A}$ & + & 0, none & -299 & . & . & 0 , none \\
\hline-248 & Db & $\mathbf{0}$ & $4,5,6,8,9$ & -300 & $\mathbf{A}$ & + & 0, none \\
\hline-249 & $\mathbf{C}$ & + & 4,5 & -301 & $\mathbf{E a}$ & . & 0, none \\
\hline-250 & $\mathbf{E a}$ & + & 0, none & -302 & D & 0 & $0,6,7,8$ \\
\hline
\end{tabular}

These secondary mutants, selected for rapid growth on L-histidinol, did not appear to be double mutants involving gene $G$ since they gave wild-type recombinants in transduction tests involving his-63 as donor (see below).

Mutant his-22 growing on L-histidinol.2HCl (30 $\mu \mathrm{g} . / \mathrm{ml}$.) in liquid medium, 
showed a slow prolonged logarithmic increase in optical density (generation time $=15-20 \mathrm{hr}$.); this was followed by a sudden shift to a faster growth rate (generation time $c .4 \mathrm{hr}$.) In contrast, on L-histidinol.2HCl (30 $\mu \mathrm{g} . / \mathrm{ml}$.) $h i s G-46$ grew logarithmically throughout at a constant rate (generation time $=2-3$ to $4-3 \mathrm{hr}$.) until culture saturation.

Histidylleucine (Dr B. N. Ames, personal communication) histidyl histidine, carnosine, histidine methyl ester, formylhistidine, $N$-acetyl-L-histidine and, to a lesser extent, thiolhistidine, imidazolelactic acid and D-histidine supported slow growth of most of the mutants. On the latter three media, fast-growing papillae usually were present. That the papillae were due to partial backmutation seems unlikely, since the clones did not plate on minimal medium and arose from many of the multisite- as well as single-site mutant strains.

Wild-type colonies completely failed to feed any of the mutant strains. This absolute failure to feed contrasts with mutants which require other amino acids (e.g. tryptophan), where sensitive methods invariably detect slight leakages of growth-promoting substances from the wild-type bacteria. Occasional prototrophic ' $h$ is $^{+}$reversions' feed all his mutants and excrete small quantities of a substance which is chromatographically identical with histidine. The genetic locus for the 'feeder' property of one such mutant is linked by transduction with the genes involving histidine biosynthesis described below. The 'feeder' strains are now being more extensivelyinvestigated,

Some of the his mutant strains fed other mutants; tests for syntrophism involving $h i s$ mutants were described by Hartman (1956). Group $D$ mutants fed mutants of all other classes but not other $D$ mutants, nor multisite mutants his-152 and -57. Group $C$ mutants fed slightly all his mutants, including themselves, other $C$ mutants, and multisite mutants such as his-152 and -57.

Hartman (1956) listed some imidazole derivatives which failed to support the growth of Salmonella his mutants. Representative mutants of each of the basic phenotypes (Table 2) also failed to grow on: spinacin; imidazole-benzylL-histidine; D-arabino-tetrahydroxybutyl-imidazole; 3-amino-1:2:4-triazole; 4-thiazole alanine 3-pyrazole alanine (Ames \& Hartman, unpublished); or on 3-methyl histidine. None of these compounds appreciably inhibited the growth of wild-type bacteria on minimal medium, and only imidazole formaldehyde at high concentrations inhibited his mutants grown on limiting L-histidine. 2-Imidazole alanine and 2-thiazole alanine did not support the growth of representative his mutants but slightly inhibited the growth of these mutants on limiting histidine and wild-type bacteria on minimal medium (Ames \& Hartman, unpublished). The degree of inhibition was grossly the same for mutants of each of the basic his groups listed in Table 2.

Growth studies thus served to distinguish several classes of mutants (Table 2). $D$ mutants failed to grow on histidinol and $G$ mutants grew most rapidly. $E$ mutants distinctively formed snaky microcolonies on minimal medium and $B, C$ and $D$ mutants failed to undergo more than one or two divisions when plated similarly. Exceptions to these general classes, usually involving 'leaky' mutants, have been noted above, and other exceptional cases will be discussed below (see 'Multisite mutations'). 


\section{Accumulations}

The patterns of accumulations are strikingly different for different mutants (Table 3). The mutants fall into definite classes in this regard (Hartman, 1956; Table 2). The largest proportion of each compound was excreted into the culture fluid rather than retained in bacteria. Contrary to an earlier report (Hartman, 1956), we failed to encounter accumulation of anthranilic acid by any of the mono-auxotrophs. Diazotizable amine, however, was accumulated, as noted for individual mutants in Table 3. Preliminary experiments have indicated that several different diazotizable amines are accumulated by certain mutants and that 'leaky' mutants, regardless of the genetic localization of the mutant site, accumulate excessive amounts of these compounds (J. C. Loper, unpublished).

\section{Efficiency of transduction}

When phage grown on wild-type bacteria was used to infect a histidine mutant, the number of wild-type recombinants recovered was directly proportional to the phage input over a wide range of multiplicities. One complete transduction was achieved for every $5 \times 10^{4}$ to $1 \times 10^{5}$ phage particles in the input. Data from an experiment with his-63 as recipient are plotted in Fig. 2, where the conditions for the experiment are outlined in the legend.

A proportion of the input was lost by lysis of a fraction of the recipient bacteria; this fraction was quite variable but always higher on EM medium than on minimal medium. Nevertheless, the yield of wild-type recombinants was nearly always equal, or several-fold greater, on EM medium as compared with minimal medium. Lysogenization by $\mathrm{P} 22$ phage was much more sensitive to histidine starvation and dependent upon the plating medium than was transduction to histidine independence. There was little or greatly delayed lysogenization on minimal medium, even when multiplicities of 5 were used. In contrast, the frequency of lysogenization/infected bacterium plated was very high on EM medium. Other experiments showed that the efficiency of transduction rapidly declined at multiplicities above 15-20 on both plating media. A great decrease in total recovery of wild-type recombinants occurred when multiplicities greater than 50-100 were used; this decrease was presumably due to lysis from without. Phage prepared on a one mutant strain always failed to yield prototrophic recombinants with that strain.

\section{Evidence for linkage of histidine mutational sites}

Reciprocal tests between histidine mutants yielded low numbers of recombinant wild-type clones as compared with transduction tests with equal numbers of phage particles grown on wild-type bacteria. This behaviour was characteristic of all such tests, involving approximately 200 different mutants, which have been completed to date. It is assumed that the deficiency in numbers of wild-type recombinants is due to the joint, or linked, transduction into the recipient cell of the donor's histidine marker together with the wildtype allele of the gene in which the recipient is defective. The increased ratio 
of abortive to complete transduction in intergenic mutant $(x)$ mutant tests, compared with mutant $(x)$ wild-type crosses, supports this contention (see Hartman et al. 1960).

The existence of multisite mutants, described below, constitutes very strong evidence that all of the his genes are linked. Convincing evidence for linkage is obtained by actual recovery of the donor genotype in the recombinant

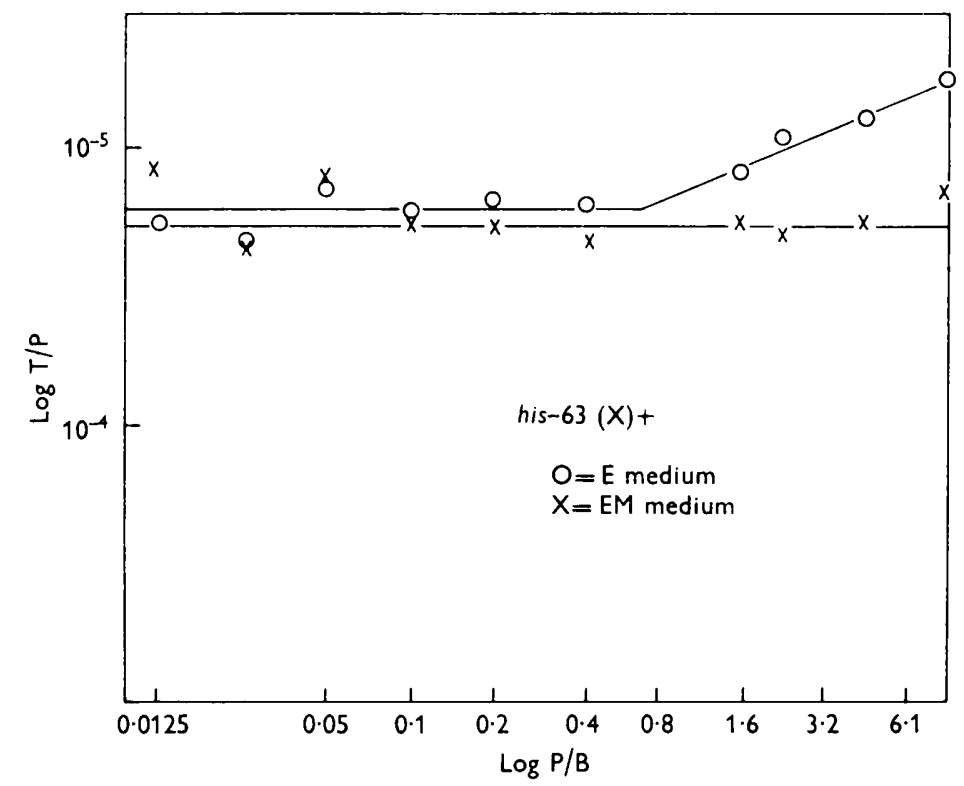

Fig. 2. The number of wild-type recombinants (complete transductions) obtained per phage particle ( $T / P)$ is plotted as a function of the phage multiplicity (average number of phage particles absorbed per bacterium $=\mathrm{P} / \mathrm{B})$. A constant number of bacteria is maintained while the phage input is varied. An $18 \mathrm{hr}$. saturated aerated nutrient broth culture of the stable mutant his- 63 was diluted $1 / 3$ in $\mathrm{T} 2$ buffer to a titre of $9.5 \times 10^{8}$ bacteria $/ \mathrm{ml}$. Samples of this suspension were mixed and left at $37^{\circ}$ for $5 \mathrm{~min}$. with equal volumes of phage suspension appropriately diluted in T2 buffer. Samples (0.1 ml.) of the mixture were spread evenly on each of 4 slightly-enriched minimal (EM) and 4 minimal (E) agar plates and incubated for 2 days at $37^{\circ}$. Wild-type recombinants (complete transductions) were scored by macroscopic observation on $\mathbf{E M}$ and on $\mathbf{E}$ plates and the minute colonies (abortive transductions) by observation of the $\mathbf{E}$ plates under a dissecting microscope. On all plates examined, the ratio of abortive to complete transduction was between 5 and 8 to 1 (not shown in figure).

clones issuing from his $(x)$ his crosses since this requires the simultaneous (joint) transduction of the mutational site of the donor together with the wildtype allele of the mutational site of the recipient, at a frequency much higher than the product of the two independent transductions. Two types of systems were used: (1) The first method takes advantage of the failure of $D$ mutants to grow on histidinol, a substance which they accumulate and excrete into the medium, thus feeding mutants of all other classes. Recipient hisD bacteria were infected with phage grown on a mutant of any other phenotypic group and plated on EM plates, additionally supplemented in some cases with 
L-histidinol ( 20 or $30 \mu \mathrm{g} . / \mathrm{ml}$.). On this medium, in addition to the wild-type recombinants which formed large colonies, small colonies characteristic of the donor phenotype were observed. Figure 2 in Hartman (1956) contains a schematic representation of this test. (2) The second type of test utilizes temperature-sensitive, 'leaky' or purine-stimulated mutants as donors and any of the other mutants as recipients. Plating was performed on minimal medium supplemented with adenine $(20 \mu \mathrm{g} / \mathrm{ml}$.$) , methionine (20 \mu \mathrm{g} . / \mathrm{ml}$.$) ,$ thiamine $(4 \mu \mathrm{g} / \mathrm{ml}$.) and pantothenate $(4 \mu \mathrm{g} / \mathrm{ml}$.). Again, the recombinant donor types appeared as small colonies and the recombinant wild-type forms as large colonies (see Pl. 1, fig. 1 in Hartman et al. 1960).

All mutants, of over 150 tested, have fulfilled the criterion of linkage by the production of bacteria of donor phenotype as mentioned above. Additional tests have confirmed the genotype of the bacteria which comprised the small colonies in a number of cases. These supplementary tests included: transduction tests with phages prepared on each of the original parents; studies of accumulations; growth characteristics; recombinational behaviour in transduction tests with other his mutants.

An occasional small colony (about $1 \%$ ) was found to contain both mutant donor-type and wild-type bacteria. This behaviour was confirmed by using the stable his-22 mutant as donor, and was probably caused by second-round mating events (cf. Demerec et al. 1958). The exact frequency of these events we cannot determine readily by the techniques at hand since most mixed clones would presumably be scored as wild-type rather than as donor type under our plating conditions.

When donors are utilized which are capable of reversion to prototrophy, the influence of mutation pressure upon the resulting proportion of large and small colonies will be most effective when very closely linked markers are used, that is, mutation to wild-type will be most disruptive when the wild-type recombinant class is very rare. Thus, the limitations imposed by spontaneous mutation must be ascertained in each case; some tests of this nature are described below.

In crosses between mutants it is the general rule that the sites of mutation of strains comprising a single phenotypic class form a very closely linked group. Experiments not reported here showed that a single-site mutation was always more closely linked with one or more other sites of its own group than it was to the most closely linked single site member of another phenotypic class. Contrariwise, a marker in one gene might appear more closely linked with markers of mutants of one other phenotypic class than it was to some of the mutants of its own phenotypic class. Thus, the genes involved in histidine synthesis are considered to be very closely linked to one another. Further, the sites of mutation which engender individual phenotypic classes are clustered together in subregions (or genes) comprising the histidine region of the chromosome. 
The relative positions of the genes

Three techniques were used in ordering the mutant sites within genes, and also the genes on a 'chromosome map' (Fig. 3).

(1) Multisite mutations. Several mutants have never been observed to mutate to prototrophy. These mutants also failed to give detectable numbers of wild-type recombinants with two or more mutants which exhibited recombination in reciprocal tests with one another. The mutants described are: his-22, $-41,-55,-63,-134,-135$ and -152 . All are derivatives of strain LT2 (Table 1). The histidine region of the chromosome is depicted in the upper part of Fig. 3; it is divided into 8 subregions, or genes, in the order $E, F, A, H$, $B, C, D$ and $G$. Strains carrying mutant sites localized in each of these genes correspond to the phenotypic classes described earlier.

Three of the genes, $E, B$ and $D$, are, in turn, subdivided into regions denoted with small letters ( $a$ and $b$, or $a, b, c$ and $d$ ) on the basis of genetic complementation (Hartman et al. 1960). Just above the upper line are arbitrary roman numeral designations for portions of the histidine chromosome segment. These denote regions which may be differentiated on the basis of genetic complementation or by the presence or absence of recombination with one or more of the multisite mutants. For example, a site of mutation is said to be located in region $\mathrm{V}$ when the strain gave no wild-type recombinants with his-135 or with his-41, but did give wild-type recombinants with his-152. A mutational site is inferred as being in region VIII when the strain yielded no wild-type recombinants in tests with his-41 or with his-55 but did give recombinants with mutants of all other regions. In analysing the mutants that are located in this manner, it was found that they have the phenotype of the proper gene (e.g. in the above examples, $F$ ). Furthermore, their basic behaviour in complementation is predictable (Hartman et al. 1960) as is their general behaviour in two-point tests with other his mutants located by similar means. Table 4 lists mutants which have been placed into the gene regions shown in Fig. 3 on the basis of failure or ability to give wild-type recombinants in crosses with various of the deletion mutants.

Multisite and stable mutations may have a propensity for 'ending' at the ends of genes. As depicted in Fig. 3, the evidence indicates that one of the two ends of his-41, both ends of his-22, and at least one end of his-63 terminate in this manner. The proportion of such cases may be higher since the present data are inadequate to exclude the possibility that the genetic lesions in several of the other mutants (e.g. his-134, -135,-203, -152) actually end at the ends of genes but inhibit recombination (for example, efficient synapsis) in adjacent regions. Contrariwise, it is impossible to exclude the possibility that multisite mutants include relatively non-essential terminal portions of the adjacent genes.

Overlapping stable mutations allow a demonstration of linear ordering of genes in the $E-A$ and $D-G$ regions (Fig. 3). Indeed, the analysis of his-63 and his-152 constituted the first definite evidence on the ordering of genes $D$ and $G$ relative to genes $B$ and $C$. 


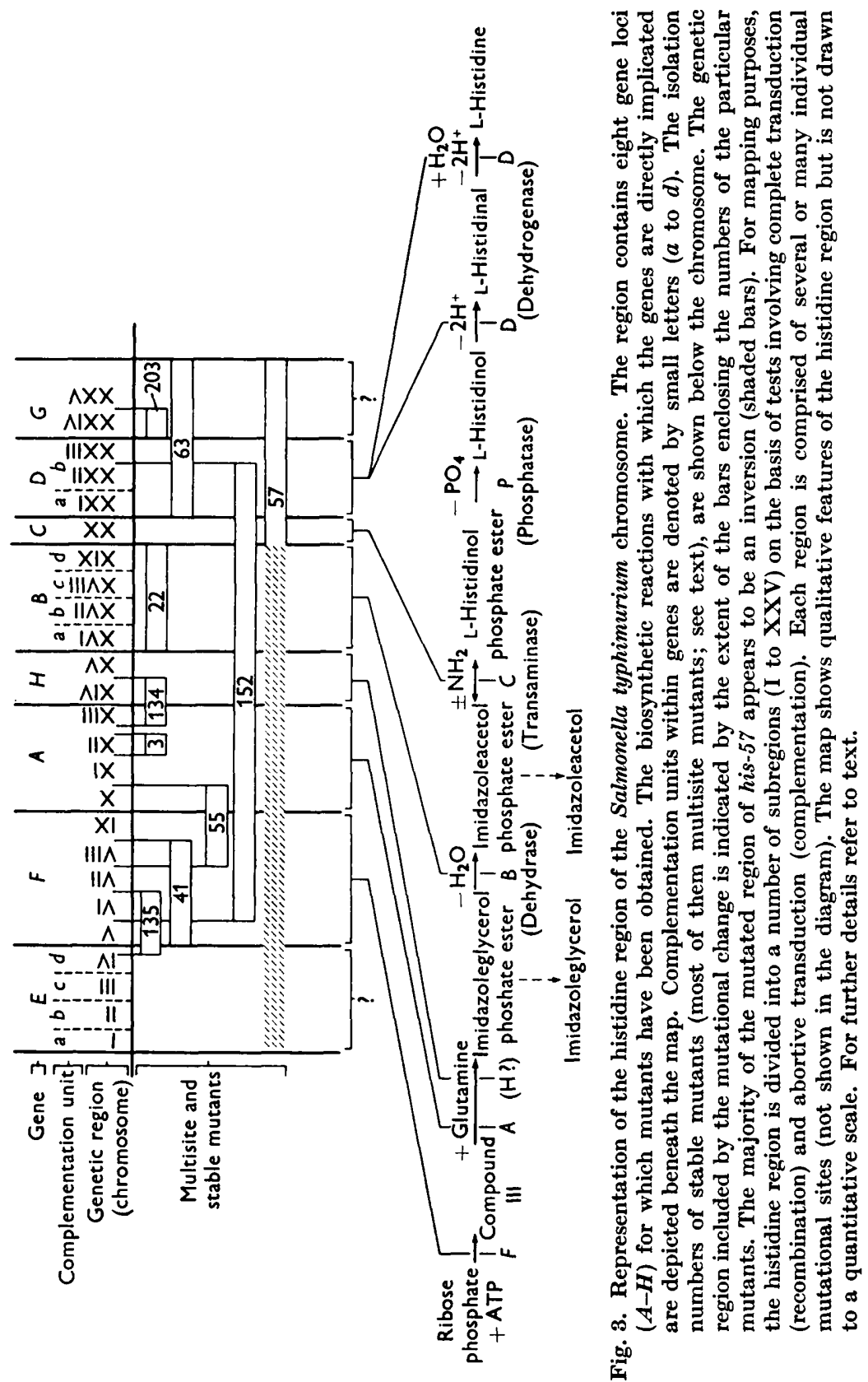


Phage grown on wild-type bacteria transduces wild-type genes into most multisite mutants with approximately the same frequency as it does into recipients carrying single-site mutations. However, a decrease of c. $75 \%$ in the number of wild-type recombinants recovered was obtained with the long multisite mutant (his-152).

Table 4. Genetic regions (designated in Fig. 3) and a list of the sites of mutation localized in each region

\begin{tabular}{|c|c|c|c|}
\hline $\begin{array}{l}\text { Class of } \\
\text { mutant, } \\
\text { or gene }\end{array}$ & Region & $\begin{array}{l}\text { Mutants listed in most } \\
\text { probable order of mutant } \\
\text { sites, from 'left' to } \\
\text { 'right' }\end{array}$ & $\begin{array}{l}\text { Other mutants with } \\
\text { sites in region specified }\end{array}$ \\
\hline $\mathbf{E}$ & $\begin{array}{l}\mathbf{I}=a \\
\mathbf{I I}=b \\
\mathbf{I I I}=c \\
\mathbf{I V}=d\end{array}$ & $\begin{array}{l}125,99 \\
284 \\
35 \\
105,145\end{array}$ & $\begin{array}{l}242,250,259,301 \\
\text { i1 } \\
\text {. }\end{array}$ \\
\hline $\mathbf{F}$ & $\begin{array}{l}\text { V } \\
\text { VI } \\
\text { VII } \\
\text { VIII } \\
\text { IX }\end{array}$ & $\begin{array}{l}58 \\
42 \\
(71,114), 45,76,44,93 \\
73,109 \\
95,81,144,6\end{array}$ & $\begin{array}{l}\cdot \\
103,148,155,158,222,224,235 \\
\text { 132, 147, 197, 198, 218, 221, } 228 \\
251,256,260,277\end{array}$ \\
\hline $\mathbf{A}$ & $\begin{array}{l}\mathbf{X} \\
\mathbf{X I} \\
\mathbf{X I I} \\
\mathbf{X I I I}\end{array}$ & $\begin{array}{l}38,80,67,69,48 \\
33,30,106,81 \\
3 \\
(134,263)\end{array}$ & $\begin{array}{l}89,100,140,153,231,294 \\
157,159,246,247,253,269 \\
\text {. } \\
\text {. }\end{array}$ \\
\hline $\mathbf{H}$ & $\begin{array}{l}\text { XIV } \\
\text { XV }\end{array}$ & $\begin{array}{l}134 \\
107,32\end{array}$ & $\dot{.}$ \\
\hline $\mathbf{B}$ & $\begin{array}{l}\text { XVI }=a \\
\text { XVII }=b \\
\text { XVIII }=c \\
\text { XIX }=d\end{array}$ & $\begin{array}{l}20,79,14,(12,24) \\
(53,206) \\
56,116,59 \\
(40,47)\end{array}$ & $\begin{array}{l}\dot{61} \\
102,136 \\
65\end{array}$ \\
\hline C & $\mathbf{x x}$ & $\begin{array}{l}43,8,50,31,201,15 \\
207,28,13,210,(2 \\
211), 209,202,62\end{array}$ & . \\
\hline D & $\begin{array}{l}\mathbf{X X I}=a \\
\mathbf{X X I I}=b \\
\mathbf{X X I I I}=b\end{array}$ & $\begin{array}{l}208,112,10 \\
(1,36,66,90), 113,18 \\
37,49,130 \\
139(54,78,133)\end{array}$ & $\begin{array}{l}\dot{27,} 34,60,68,83,85,216 \\
150\end{array}$ \\
\hline G & $\begin{array}{l}\text { XXIV } \\
\text { XXV }\end{array}$ & $\begin{array}{l}70,46,200 \\
119,205,204,52,72\end{array}$ & $\begin{array}{l}216, \\
199,255\end{array}$ \\
\hline
\end{tabular}

Attempts were made to obtain incorporation of only a portion of the multisite regions in his-63 and his-152. Phage was grown on wild-type bacteria and selection was performed on histidinol-containing medium. No transduction of his-163 and his-152 recipients was observed to occur to $h i s^{-}$hisD $^{+}$; all recombinants were wild-type. Thus, if present, transduction to his $^{-}$his $^{+}$ occurred with less than $\mathbf{0 . 3 0} \%$ of the frequency of transduction to the wildtype phenotype.

Hartman (1956) reported that his $H-32$ produced, with extremely low frequency, wild-type recombinants in tests with the multisite mutant hisB-22. 
More extensive tests have demonstrated that large ('wild-type') colonies arose only when hisH-32 was used as donor under conditions which allowed growth of the donor-type clones into small colonies. Reconstruction experiments (similar to those described below 'Ratio test') demonstrated similar proportions of large (spontaneous reversions to prototrophy of hisH-32) and small (his $H-32$ ) colonies under analogous plating conditions. Thus there now remains no evidence for recombination between the his $\mathrm{H}-32$ and hisB-22 markers. Inability to yield wild-type recombinants in this combination is surprising in view of the complementation observed between hisH-32 and $B$-22 (Hartman et al. 1960) and the dissimilar biochemical phenotypes of the two mutants (Table 3). All $C$ mutants produced wild-type recombinants with his-22. A similar situation existed with regard to multisite mutation hisD, $G-63$ which: (1) was unable to give wild-type recombinants in tests with hisC-62, C-202 and $C$-209; (2) yielded recombinants with all other $C$ mutants tested (those listed in Table 3); (3) weakly complemented all except $D$ and $G$ mutants (Hartman, 1960); (4) failed to accumulate detectable Pauly positive substances. The chromosome regions marked off in Fig. 3 for his-22 and his-63 take into consideration the complementation behaviour of these two mutants. The failure of recombination of his-22 with his-32 indicates that genes $H$ and $B$ are adjacent; similarly, the data cited involving his-63 indicate that gene $C$ is adjacent to gene $D$. The genetic sequence proposed is depicted in Fig. 3.

Mutant his-57 is non-transducible and has never been detected to revert to wild-type. As donor in transduction tests, however, his-57 engendered wildtype recombinants with all $E$ and $F$ strains and, with generally decreasing frequency, with most $A, H$ and $B$ strains tested. These tests included the formation of wild-type recombinants in crosses with the stable or multisite mutants his-135, -41 , and -134 and with many $h i s B$ strains. While recombinants were produced in crosses with many single-site $B$ mutants, no wild-type recombinants were elicited from reciprocal tests with $h i s-22$ or in tests with $C, D$ and $G$ recipients (see Fig. 3). The growth of his-57, like all other histidine mutants studied, was completely restored with L-histidine. Genes contained in phage prepared on wild-type bacteria weakly complemented his-57 (very small minute colony size). Complementation was not detected with his-57 used as donor or as recipient in combination with any his mutant (Hartman et al. 1960). No Pauly or Bratton-Marshall accumulations were found for his-57 nor would it grow on histidinol (Table 1). Mutant his-57 was deficient in all four enzyme activities examined (Ames et al. 1960).

(2) 'Best-fit' method. In crossing a number of histidine mutants in all possible combinations it was expected that the number of wild-type recombinants on minimal medium would indicate the distance between the mutational sites involved, those further apart producing relatively more recombinants. Only a single order of the mutational sites should satisfy each of the individual results obtained when 'point' mutations are used in the analysis if the frequency of recombination is proportional to the distances between two markers. Data obtained from this type of experiment have been 
published and a linkage map constructed (table 1 and fig. 1 in Hartman, 1956).

Many sources of error are inherent in the method: (1) a large number of independent variables influence absolute transduction frequencies (phage titre, phage transducing potency, multiplicity of infection, plating conditions, physiological state of the plating organisms, mode of incubation, etc.); (2) different alleles exert specific effects on recombination probabilities (discussed below). The method is accurate, nevertheless, when the markers involved in the tests are distributed over a comparatively long region of the chromosome, for example, in different genes, and when all technical factors are carefully controlled. The data from a large number of such experiments, not presented here, strongly support the order of the genes as depicted in Fig. 3. The method is only indicative in the analysis of short regions, that is, in ordering mutational sites within single genes or parts of the genes. The orders for sites within genes given in Table 4 were mainly derived in this manner and are, therefore, only approximations.

One his mutant, in transduction tests with phage grown on another his mutant, may produce as many as $50-60 \%$, or as low as about $0.1 \%$, of the number of wild-type recombinants obtained with the same number of equally potent phage particles grown on wild-type bacteria. Beyond this low value, the formation of wild-type recombinants ceased, although our methods are theoretically sensitive enough to detect recombination 100 times less frequent. Failure to obtain recombinants, in spite of extensive testing, was noted in the following combinations: (1) hisD-1, -36, -66, -90; (2) hisD-9, -18; (3) hisD54, -78, -133; (4) hisF-71, -114; (5) hisB-40, -47; (6) hisB-12, -24; (7) hisB-53, $-206 ;(8)$ hisC $-2,-211$. Mutation of each of these strains to the wild-type phenotype was observed, and each strain so far has been determined as including only a single mutational site. All the members of each of the individual 'sets' of mutations had identical biochemical phenotypes, appeared to be localized in the same place in relation to other mutations (i.e. at the same site within a gene locus as determined by recombination tests with, for example, non-allelic mutants; see Fig. 3). All are of the same complementation type except hisBab-53 and hisBb-206 (Hartman et al. 1960). Members of the sets may not be identical in other accessory respects, such as: rate of mutation to prototrophy (P. E. Hartman, unpublished; Kirchner, unpublished) and in recombinational behaviour when crossed with other mutants (see below).

(3) Ratio test. Donor-type and wild-type recombinants can be recovered on the same plates. This provides a test, with some internal control, for the positioning of mutant sites by the determination of the probability $(\bar{p})$ of individual integration as opposed to the total number of recombinants (individual + joint integrations). Thus $\bar{p}$ indicates the probability, per recombinational event through which integration of one marker takes place, that it will be integrated separately from a second closely linked marker. The value obtained for $\bar{p}$ in a particular test remained relatively independent of the plating medium as long as the medium allowed a sufficient amount of growth 
to permit quantitative enumeration of the donor-type colonies. For example, in one experiment, phage produced on his-107 yielded, upon infection of his-46, the following proportions of wild-type recombinants as compared with total recombinants: (1) on $E$ medium, 0.38 ; (2) on EM medium, 0.30 ; (3) on E medium supplemented with adenine + thiamine + methionine + pantothenate, $0 \cdot 33 ;(4)$ on EM medium similarly supplemented, $0 \cdot 29$. In repeated tests performed on a uniform medium, the variation in proportions of wildtype to total colonies was always the same as, or less than, that obtained above. Precautions must be taken to count significant numbers of the minor recombinant type (wild-type), to avoid crowding of colonies of the plates, and to assure uniform detection of the small donor-type colonies. Usually 100-300 total colonies were scored per plate. The presence of a number of rounds of 'mating' between the host chromosome and transduced fragment (Demerec et al. 1958) will also have little effect on the values obtained for $\bar{p}$ because of the plating technique whereby the minority component is similarly scored, whether or not the majority recombinant type is simultaneously present in the clone. Furthermore, second-round matings appear to produce the same relative proportions of recombinant types as do firstround matings (Demerec et al. 1958).

Mutation to wild-type during growth of the small colonies did not greatly influence the ratios obtained in the combinations used in our study. This was determined by : (1) reconstruction plating experiments of uninfected and phageinfected bacteria; (2) transduction into multisite mutants (e.g. his-56 into his-152 or his-22); (3) tests involving extremely closely linked mutant sites where linkage is compared with conditions under which there is no growth of donor type transductional clones; (4) actual identification of the genotype of the donor strain. The possibility remains that the mutant allele in some cases was more mutable when present in the genetic background afforded by the recipient genome. In ratio tests, only the more stable of the histidine mutants were used as recipients, thus minimizing any possible influence of residual genotype on mutation of the his genes. Two strains, his-12 and his-24, each mutated to prototrophy at comparable rates before and after placement, by joint transduction, into his-1.

The experimental value obtained for $\bar{p}$ depended first of all upon the specific combination of mutants tested. For example, two mutants, his-24 and his-12, engendered no wild-type recombinants in reciprocal tests with one another. In combinations with all other mutants, however, his-24 always exhibited a two-to-fourfold higher value for $\bar{p}$ than did his-12. The two mutations were placed, by joint transduction, into a culture of his-1 newly arising from a single colony isolate; two clones of each donor-type were isolated. Each of the clones retained the behaviour in recombination typical of the donor strain. Furthermore, various reconstruction experiments showed no appreciable difference between $h i s-12$ and $h i s-24$ when plated on several different recipient strains either in the absence of phage infection or after infection with phage prepared on his-152 (which elicited no detectable recombinants from any of the strains involved in the tests). The total number of recombinants obtained with his-24 
was generally 25-50\% higher than the number obtained with his-12 as recipient.

The fragmentary nature of the genetic material transduced (Lederberg, 1955; Demerec, $1956 b$ ) and the action of specific alleles on recombination frequencies make it impossible to assign classical recombination values for the histidine region of the Salmonella chromosome. The latter effect also rules out

Table 5a. Mean probability of independent integration $(\bar{p})$ is shown for five recipients mutant in locus $D$ in tests involving mutants at 7 other loci as donors

The number columns (No.) indicate the number of different alleles of the locus utilized in the tests.

\begin{tabular}{|c|c|c|c|c|c|c|c|c|c|c|c|c|c|c|}
\hline \multicolumn{3}{|c|}{$\begin{array}{l}\text { Loci mutant } \\
\text { in donor... } \\
\text { Recip- }\end{array}$} & \multicolumn{2}{|r|}{$\mathbf{F}$} & \multicolumn{2}{|r|}{$\mathbf{A}$} & \multicolumn{2}{|r|}{$\mathbf{H}$} & \multicolumn{2}{|r|}{ B } & \multicolumn{2}{|r|}{ C } & \multicolumn{2}{|r|}{ G } \\
\hline ient & No. & $\overline{\boldsymbol{p}}$ & No. & $\bar{p}$ & No. & $\bar{p}$ & No. & $\bar{p}$ & No. & $\bar{p}$ & No. & $\bar{p}$ & No. & $\bar{p}$ \\
\hline$h i s D-49$ & 1 & $0 \cdot 382$ & 2 & 0.308 & 2 & $0 \cdot 219$ & 2 & $0 \cdot 165$ & 2 & $0 \cdot 193$ & 24 & $0 \cdot 143$ & 8 & 0.074 \\
\hline D-208 & 6 & 0.421 & 5 & 0.361 & 4 & 0.259 & 2 & $0 \cdot 101$ & 11 & $0 \cdot 189$ & 24 & 0.089 & 8 & 0.144 \\
\hline$D-1$ & 4 & $0 \cdot 315$ & 17 & $0 \cdot 290$ & 15 & 0.260 & 1 & 0.164 & 21 & $0 \cdot 172$ & 24 & 0.083 & 8 & 0.077 \\
\hline D. 18 & 2 & 0.458 & 3 & 0.419 & 4 & $0 \cdot 370$ & 1 & 0.251 & 8 & $0 \cdot 276$ & 24 & 0.149 & 8 & 0.091 \\
\hline$D-10$ & 2 & $0 \cdot 252$ & 4 & $0 \cdot 243$ & 4 & $0 \cdot 185$ & 1 & 0.046 & 8 & $0 \cdot 177$ & 24 & 0.072 & 8 & 0.077 \\
\hline Mean & . & $0 \cdot 366$ & . & $0 \cdot 324$ & . & $0 \cdot 259$ & . & $0 \cdot 157$ & . & $0 \cdot 210$ & . & $0 \cdot 107$ & . & 0.093 \\
\hline
\end{tabular}

Table $5 b$. Mean probability of independent integration $(\bar{p})$ is shoron for recipients of each locus in tests involving each of 9 strains serving as donors

The number columns (no.) indicate the number of different alleles of the locus utilized in the tests.

\begin{tabular}{|c|c|c|c|c|c|c|c|c|c|c|c|c|c|c|c|c|c|c|}
\hline \multirow{2}{*}{$\begin{array}{c}\text { Donor } \\
\text { Recip- } \\
\text { ient }\end{array}$} & \multicolumn{2}{|c|}{ his $A-80$} & \multicolumn{2}{|c|}{$A-48$} & \multicolumn{2}{|c|}{$A-106$} & \multicolumn{2}{|c|}{$H-107$} & \multicolumn{2}{|c|}{$\mathrm{H}-32$} & \multicolumn{2}{|c|}{$\mathrm{H}-32$} & \multicolumn{2}{|c|}{$B-56$} & \multicolumn{2}{|c|}{$B-116$} & \multicolumn{2}{|c|}{$C-15$} \\
\hline & No. & $\overline{\boldsymbol{p}}$ & No. & $\bar{p}$ & No. & $\bar{p}$ & No. & $\overline{\boldsymbol{p}}$ & No. & $\overline{\boldsymbol{p}}$ & No. & $\bar{p}$ & No. & $\vec{p}$ & No. & $\bar{p}$ & No. & $\overline{\boldsymbol{p}}$ \\
\hline $\mathbf{E}$ & 4 & $0 \cdot 167$ & 4 & 0.251 & & & 4 & $0 \cdot 109$ & 4 & 0.078 & 4 & $0 \cdot 163$ & . & • & 4 & 0.082 & 4 & 0.283 \\
\hline $\mathbf{F}$ & 4 & $0 \cdot 189$ & . & . & 4 & $0 \cdot 144$ & 5 & $0 \cdot 250$ & 5 & 0.122 & 7 & $0 \cdot 154$ & 7 & $0 \cdot 240$ & 5 & 0.228 & 7 & 0.224 \\
\hline $\mathbf{A}$ & . & - & . & 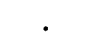 & 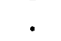 & 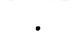 & 4 & $0 \cdot 128$ & 4 & $0 \cdot 124$ & 4 & $0 \cdot 114$ & 4 & 0.214 & 4 & 0.229 & 3 & 0.261 \\
\hline H & . & • & . & - & $\cdot$ & . & 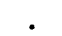 & & . & 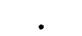 & 1 & 0.053 & . & 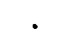 & . & . & . & . \\
\hline B & 9 & 0.350 & 4 & $0 \cdot 127$ & 6 & $0 \cdot 181$ & 9 & $0 \cdot 142$ & 12 & 0.058 & 12 & 0.028 & 8 & 0.033 & 10 & $0 \cdot 083$ & 9 & $0 \cdot 120$ \\
\hline C & 1 & 0.368 & 1 & $0 \cdot 195$ & • & . & 2 & $0 \cdot 169$ & 4 & 0.093 & 2 & $0 \cdot 151$ & 2 & 0.076 & 2 & 0.075 & 2 & 0.046 \\
\hline D & 3 & 0.521 & $\mathbf{3}$ & 0.216 & 2 & 0.289 & 5 & $0 \cdot 165$ & 10 & $0 \cdot 165$ & 4 & $0 \cdot 151$ & 1 & $0 \cdot 117$ & $\mathbf{3}$ & 0.079 & 11 & 0.144 \\
\hline G & 5 & 0.500 & 5 & 0.517 & 5 & 0.553 & 5 & 0.387 & 5 & 0.354 & 4 & 0.391 & 2 & $0 \cdot 234$ & 5 & 0.356 & 5 & 0.433 \\
\hline
\end{tabular}

the use of a simple two-point test for the unambiguous determination of relative map positions. However, we can express comparative recombination frequencies in the histidine region by the use of average values for a single mutant in tests with several other mutants closely linked to one another. This procedure tends to randomize the deviations due to individual recombinational behaviour. Tables $5 a$ and $5 b$ present mean recombination probabilities expressed in this fashion. In Table $5 a$, several $D$ mutants were used as recipients in tests with mutants from the seven other genes. In Table $5 b$, nine leaky or temperature-sensitive mutants were used as donors and recombination was studied with a number of strains mutant within or outside of their respective genes. 
The ordering of the genes by the average probability of individual integration is largely consistent with the order dictated by multisite mapping and by the 'best-fit' method based solely on numbers of wild-type recombinants. The number of strains tested in most individual cases was low, however, so that the effects of individual alleles or recombination frequencies was not entirely overcome in the pooled data. This is particularly true, in Table $5 a$, for gene $H$ donors; only two alleles were available and both gave recombinational frequencies lower than expected (hisH-32 being much lower than hisH-107).

Mutant his-211 was obtained from hisC-2 culture as a slow growing and temperature-sensitive 'back-mutant'. With his-2 as recipient, phage prepared on $h i s-211$ produced exclusively donor-type recombinants. When tested with a number of other mutants, his-211 mapped as mutant between genes $B$ and $D$ (i.e. in gene $C$ ). Only his-211 was recovered after joint transduction, for example, into a $D$ strain recipient under conditions theoretically sensitive enough to detect one his-2 recombinant out of $c$. 750 donor-type colonies

Table 6. Total number of colonies analysed and probability of independent integration $(p)$ are shown for 12 hisD recipients in tests involving 24 hisC and 8 hisG donors

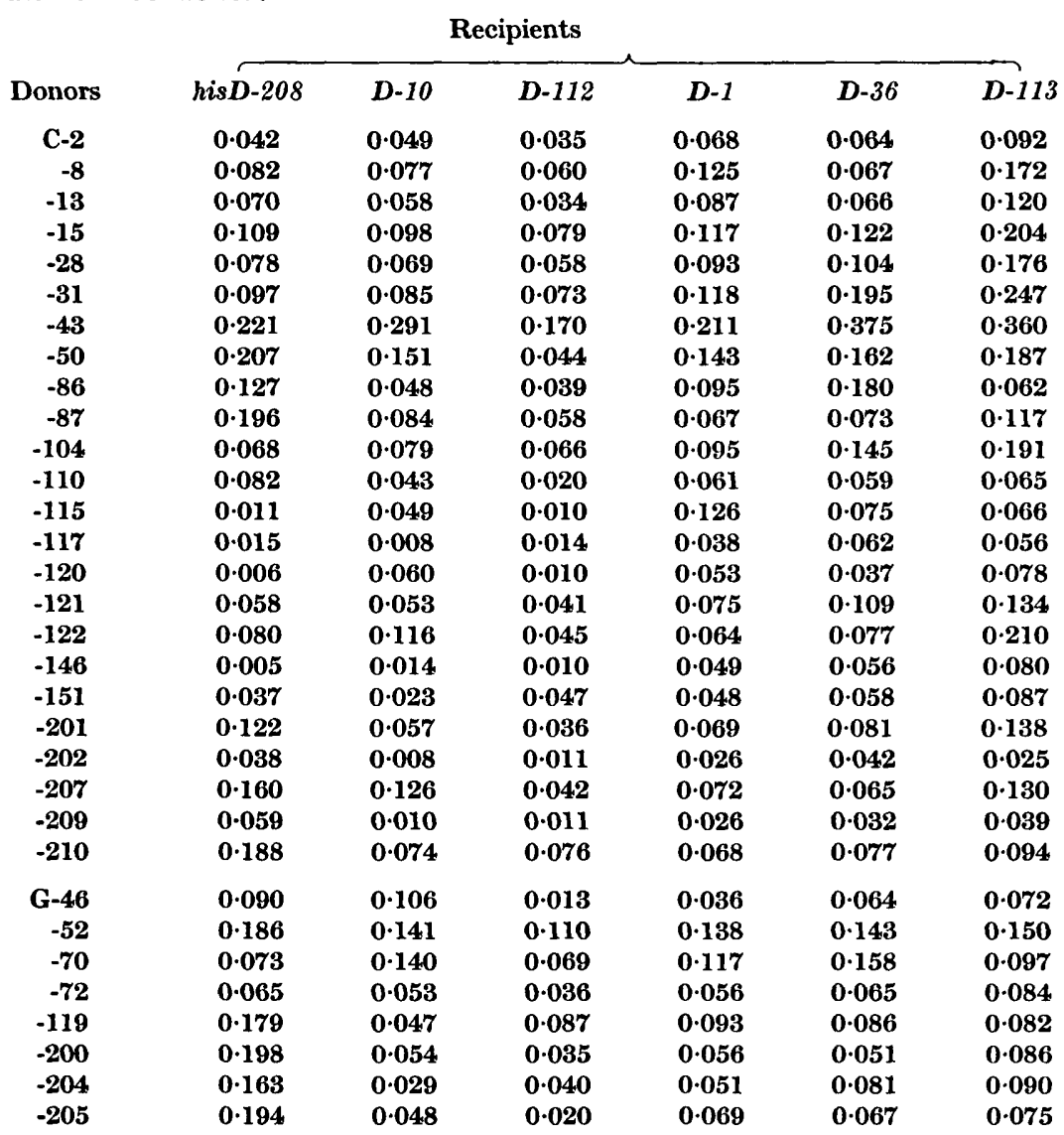


Table 6 (cont.)

\begin{tabular}{|c|c|c|c|c|c|c|}
\hline \multirow[b]{2}{*}{ Donors } & \multicolumn{6}{|c|}{ Recipients } \\
\hline & hisD-18 & $D-49$ & $D-37$ & $D-133$ & $D-130$ & $D-9$ \\
\hline $\mathrm{C}-2$ & $0 \cdot 150$ & $0 \cdot 063$ & $0 \cdot 105$ & 0.082 & $0 \cdot 151$ & 0.238 \\
\hline-8 & $0 \cdot 153$ & 0.095 & $0 \cdot 151$ & $0 \cdot 149$ & $0 \cdot 204$ & $0 \cdot 252$ \\
\hline-13 & $0 \cdot 154$ & 0.094 & $0 \cdot 116$ & $0 \cdot 115$ & $0 \cdot 169$ & $0 \cdot 332$ \\
\hline-15 & $0 \cdot 180$ & $0 \cdot 123$ & $0 \cdot 152$ & $0 \cdot 176$ & $0 \cdot \mathbf{2 2 8}$ & 0.401 \\
\hline-28 & $0 \cdot 144$ & 0.078 & $0 \cdot 133$ & $0 \cdot 145$ & $0 \cdot 133$ & $0 \cdot 307$ \\
\hline-31 & $0 \cdot 171$ & $0 \cdot 135$ & $0 \cdot 183$ & $0 \cdot 125$ & $0 \cdot 157$ & $0 \cdot 300$ \\
\hline-43 & $0 \cdot 292$ & $0 \cdot 247$ & $0 \cdot 569$ & $0 \cdot 324$ & $0 \cdot 294$ & $0 \cdot 609$ \\
\hline-50 & $0 \cdot 198$ & $0 \cdot 179$ & $0 \cdot 166$ & $0 \cdot 301$ & $0 \cdot 169$ & 0.404 \\
\hline-86 & $0 \cdot 163$ & $0 \cdot 127$ & $0 \cdot 137$ & $0 \cdot 327$ & $0 \cdot 309$ & $0 \cdot 220$ \\
\hline-87 & $0 \cdot 190$ & 0.250 & $0 \cdot 151$ & $0 \cdot 207$ & $0 \cdot 242$ & $0 \cdot 275$ \\
\hline-104 & $0 \cdot 186$ & $0 \cdot 139$ & $0 \cdot 130$ & $0 \cdot 167$ & $0 \cdot 265$ & $0 \cdot 278$ \\
\hline-110 & $0 \cdot 193$ & 0.075 & 0.082 & $0 \cdot 104$ & $0 \cdot 131$ & $0 \cdot 224$ \\
\hline-115 & $0 \cdot 133$ & $0 \cdot 131$ & 0.082 & $0 \cdot 303$ & $0 \cdot 150$ & 0.223 \\
\hline-117 & $0 \cdot 149$ & $0 \cdot 105$ & 0.073 & $0 \cdot 146$ & $0 \cdot 056$ & $0 \cdot 126$ \\
\hline-120 & $0 \cdot 123$ & $0 \cdot 154$ & $0 \cdot 024$ & $0 \cdot 211$ & $0 \cdot 133$ & $0 \cdot 118$ \\
\hline-121 & $0 \cdot 202$ & $0 \cdot 196$ & $0 \cdot 122$ & $0 \cdot 201$ & $0 \cdot 333$ & $0 \cdot 190$ \\
\hline-122 & $0 \cdot 239$ & $0 \cdot 168$ & $0 \cdot 131$ & $0 \cdot 184$ & 0.276 & $0 \cdot 313$ \\
\hline-146 & $0 \cdot 145$ & $0 \cdot 107$ & $0 \cdot 132$ & $0 \cdot 163$ & 0.083 & $0 \cdot 308$ \\
\hline-151 & 0.092 & $0 \cdot 070$ & 0.088 & $0 \cdot 106$ & $0 \cdot 126$ & $0 \cdot 174$ \\
\hline-201 & $0 \cdot 211$ & $0 \cdot 136$ & $0 \cdot 129$ & $0 \cdot 157$ & $0 \cdot 126$ & $0 \cdot 354$ \\
\hline-202 & $0 \cdot 101$ & 0.071 & 0.044 & $0 \cdot 141$ & $0 \cdot 157$ & $0 \cdot 112$ \\
\hline-207 & $0 \cdot 250$ & $0 \cdot 163$ & $0 \cdot 106$ & $0 \cdot 156$ & 0.220 & 0.351 \\
\hline-209 & $0 \cdot 135$ & $0 \cdot 138$ & 0.094 & $0 \cdot 168$ & 0.273 & $0 \cdot 145$ \\
\hline-210 & $0 \cdot 227$ & $0 \cdot 395$ & $0 \cdot 272$ & $0 \cdot 217$ & $0 \cdot 280$ & 0.297 \\
\hline G-46 & 0.078 & 0.031 & 0.044 & 0.040 & 0.029 & $0 \cdot 142$ \\
\hline-52 & 0.091 & $0 \cdot 153$ & $0 \cdot 128$ & 0.047 & $0 \cdot 159$ & $0 \cdot 206$ \\
\hline-70 & $0 \cdot 223$ & 0.073 & $0 \cdot 103$ & 0.085 & $0 \cdot 167$ & $0 \cdot 260$ \\
\hline-72 & 0.070 & 0.022 & 0.049 & $0 \cdot 043$ & 0.050 & 0.071 \\
\hline-119 & $0 \cdot 154$ & 0.099 & $0 \cdot 151$ & $0 \cdot 049$ & 0.031 & $0 \cdot 128$ \\
\hline-200 & $0 \cdot 188$ & $0 \cdot 104$ & 0.085 & 0.039 & $0 \cdot 105$ & $0 \cdot 175$ \\
\hline-204 & $0 \cdot 155$ & 0.067 & 0.026 & 0.035 & 0.085 & $0 \cdot 122$ \\
\hline-205 & $0 \cdot 152$ & 0.046 & $0 \cdot 068$ & 0.024 & 0.006 & $0 \cdot 227$ \\
\hline
\end{tabular}

tested. Furthermore, the mutant his-211 exhibited as donor a much higher proportion of wild-type recombinants (i.e. a much higher value for $\bar{p}$ ) with all other non-identical mutants tested than did his-2.

\section{Order of mutant sites within single genes}

Table 6 presents data for individual tests of $D$ mutants, as recipients, with 24 $C$ mutants and $8 G$ mutants as donors. The data for some combinations are the summed data of several experiments. Variation between experiments generally did not exceed $30 \%$ of the value shown when significant numbers of the minor (wild-type) recombinant class were obtained. The mean probabilities for recombination separating the $D$ mutants from the $C$ and $G$ mutants are presented in Table 7 . The ratio of these two values is presumed to give an indication of the order of mutant sites (fourth column in Table 7). In the fifth column of Table 7 is a calculation which adjusts all of the recombination values to common terms (i.e. equalizes the relative map distance from the average C mutant). 
The map obtained is depicted in Fig. 4. The pairs of mutants, his-1 and his36 , and his-9 and his-18 which were unable to transduce one another to wildtype in reciprocal tests, map very close to one another by the calculations derived from crosses to outside markers. Only four $D$ mutants gave wild-type

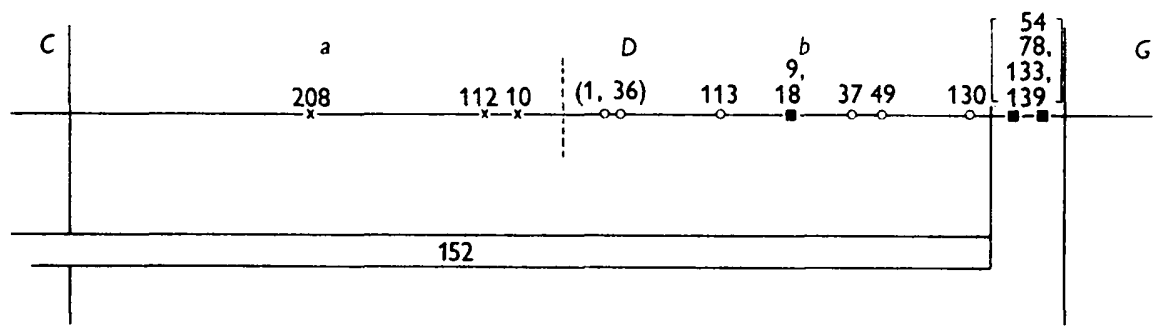

Fig. 4. Map, drawn to scale and showing the relative locations of 15 mutant sites within the hisD (histidinol dehydrogenase) locus of Salmonella typhimurium. Complementation reactions are described in Hartman et al. (1960); other details in the text. $\times$, Complementation pattern $a$; $O$, complementation pattern $b ; \square$, complementation pattern ab.

Table 7. Summary of data in Table 6 and its use in positioning of mutant sites within the hisD region of Salmonella typhimurium

For details, consult the text.

\begin{tabular}{|c|c|c|c|c|}
\hline Mutant & $\begin{array}{c}\text { Mean } \bar{p} / C \\
\text { mutants }\end{array}$ & $\begin{array}{l}\text { Mean } \bar{p} G \\
\text { mutants }\end{array}$ & Ratio: $\bar{p} C / \bar{p} G$ & Ratio: $\frac{p C / p G}{1+p C / p G}$ \\
\hline his-208 & 0.089 & $0 \cdot 144$ & $0 \cdot 62$ & 0.383 \\
\hline-112 & $0 \cdot 045$ & $0 \cdot 051$ & 0.88 & 0.468 \\
\hline-10 & 0.072 & 0.077 & 0.93 & 0.482 \\
\hline-1 & 0.083 & 0.077 & $1 \cdot 08$ & 0.519 \\
\hline-36 & 0.099 & 0.089 & $1 \cdot 11$ & 0.526 \\
\hline-113 & $0 \cdot 130$ & 0.092 & $1 \cdot 42$ & 0.587 \\
\hline-9 & 0.273 & $0 \cdot 166$ & $1 \cdot 64$ & 0.621 \\
\hline-18 & $0 \cdot 149$ & 0.091 & 1.64 & $0 \cdot 621$ \\
\hline-37 & $0 \cdot 140$ & 0.074 & $1 \cdot 89$ & $0 \cdot 654$ \\
\hline-49 & $0 \cdot 143$ & 0.074 & 1.93 & 0.659 \\
\hline-130 & $0 \cdot 190$ & 0.079 & $2 \cdot 41$ & 0.707 \\
\hline
\end{tabular}

recombinants with his-152; they are included in Fig. 5 on this basis. Of these four none was stable enough for precise ratios to be computed. The map depicted in Fig. 4 is considered to more accurately present the relationships of the mutant sites in gene $D$ than does one previously constructed (Hartman, 1956) which was based on more scanty data obtained solely on the numbers of wild-type recombinants in reciprocal tests between $h i s D$ alleles.

The data in Table 6 also may be used to determine whether there is a strain influence (LT-2 or LT-7; see Table 1) on the values obtained for $\bar{p}$. Ten LT-2 recipients and two LT-7 recipients were used in combination with 14 $C$ mutants derived from LT-2 and $10 C$ mutants from LT-7. The following average values for $\bar{p}$ were obtained: LT-2 $(\times)$ LT-2, 0.152; LT-2 ( $\times)$ LT-7. 0.097; LT-7 $(\times)$ LT-7, 0.076; LT-7 $(\times)$ LT-2, 0.135. There thus appears no lowering of $\bar{p}$ in interstrain as opposed to intrastrain combinations.

Data, obtained in a fashion similar to that used for Table 6, was analysed 
for three $B c$ mutants $(B c-56,-59$ and -116$)$ as donors and various $B$ mutant recipients. Calculations from the limited data give the following average $\bar{p}$ value for recombination with $B c$ donors: $B a, 0.085$ (average of 12 values), $B b, 0.038$ (average of 9 values), $B c, 0.015$ (average of 3 values), $B d 0.042$ (average of 6 values). The limitations in the data and the effects of individual alleles on recombination frequencies disallow precise calculations of the relative orders of individual mutants at the present time within locus $B$. A tentative order, consistent with the results to date, is listed in Table 4.

\section{DISCUSSION}

\section{Classes of his mutants and the functions of his genes}

Most of the his mutants may be classified into one of the seven general phenotypic classes listed in Table 2. Some of the classes are separable by growth properties alone; the separation of other classes requires analyses for accumulations. Still other classes are not separated until analyses extend to genetic complementation and the cell-free enzyme level. Genetic tests demonstrate that the members of each of the phenotypic classes result from genetic damage in a restricted portion (gene locus) of the $h i s$ region of the Salmonella chromosome. Genes $B, C$ and $D$ have been shown to be clearly associated with specific blocks in the biosynthetic pathway (Ames et al. 1960) as depicted in Fig. 3. Loci $\boldsymbol{F}$ and $\boldsymbol{A}$ have been implicated as involving two consecutive early steps in histidine synthesis, $\boldsymbol{F}$ in the formation of compound III and $\boldsymbol{A}$ in the conversion of compound III to imidazoleglycerol phosphate ester (Moyed \& Magasanik, 1957; Magasanik, 1958; and personal communication). Strains classified as $\boldsymbol{A}$ and $\boldsymbol{H}$ mutants are phenotypically identical in experiments performed to date, but are separable on the basis of behaviour in complementation tests (Hartman et al. 1960). In Fig. $3 \mathrm{H}$ is assumed to be concerned with a second reaction between compound III and imidazole glycerol phosphate ester (cf. Moyed \& Magasanik, 1958). However, it is equally possible that $\boldsymbol{H}$ mutants represent alleles of a second complementation unit of gene $\boldsymbol{A}$ (Hartman et al. 1960).

The functions of genes $E$ and $G$ are unknown. Mutants of these loci have the following properties: (1) specifically require L-histidine, or compounds presumably giving rise to L-histidine, for growth; (2) do not show sparing of this histidine requirement by purines, glutamic acid or glutamine; (3) show a growth response on limiting L-histidine quantitatively similar to that of mutants of other loci; (4) fail to feed other mutants or to accumulate or excrete Pauly-positive substances; (5) metabolize L-histidine to amounts of urocanic acid comparable to that obtaining for other his mutants (Hartman, 1956; Šerman, unpublished); (5) show all the enzyme activities which have been examined for in cell-free extracts (see Table 2); (7) grow on L-histidinol; (8) do not appear to be hypersensitive in vivo to enzyme repression (Ames \& Gary, 1959) or to histidine analogues (cf. Moyed \& Friedman, 1959). In addition, a feature of $G$ mutants is their comparatively rapid growth on Lhistidinol. Major unique characteristics of $\mathbf{E}$ mutants are their high efficiency 
in complementation (Hartman et al. 1960) and their growth following exhaustion of histidine from the medium. L-Histidinol phosphate ester phosphatase activity has not been detected in extracts of some multisite his mutants (Ames et al. 1960), suggesting the possibility that the gene for the phosphatase may be located in the his region of the chromosome. However, no mutants uniquely deficient in this activity have been found in Salmonella.

Structure of the his region of the Salmonella chromosome

The data indicate that the order of at least five histidine genes on the linkage map parallels the sequence of biochemical reactions (Ames et al. 1959) in the primary pathway of L-histidine synthesis (Fig. 3). Other cases of such gene arrangements in Salmonella were discussed in a recent review by Demerec \& Hartman (1959). The ordering of the his genes has been accomplished by three methods which produce results consistent with one another: (1) the presence or absence of recombination with a series of multisite mutations; (2) the numbers of wild-type recombinants resulting from two-point tests; (3) the average probability of individual integration of one marker in combinations involving a series of other, closely linked markers. Although multisite mutants have been of especial value in the analysis, no single one of these methods is considered sufficient in itself for ordering of all of the genes: it is the consistency of permissible interpretation between the three methods which has established the sequence depicted in Fig. 3. The genes appear to be discrete, non-overlapping entities arranged in a linear order. The data indicate that the his genes are truly, or very nearly, adjacent to one another.

\section{Structure of the hisD gene}

In Tables 6 and 7 data are presented, and in Fig. 4 a map is constructed, for gene $D$. We may assume: $(a)$ that the mutational sites of $C$ and $G$ donor strains are randomly distributed in recombinational behaviour and in position within each of the two genes; $(b)$ that there is equal opportunity for recombination to the 'left' of $C$ and to the 'right' of $G$. The prediction may be made that $D$ mutants must map within the central 0.500 units (from 0.250 to 0.750 ) if the genes are of equal size. This has been the case, and the limits to gene $D$ are so indicated to scale in Fig. 4. The $D$ mutants are divided into three functional classes on the basis of complementation patterns (Hartman el al. 1960). The recombinational analysis shows that mutational sites of mutants of each of the two basic complementation classes ( $a$ and $b$ ) are grouped. Thus, the gene is divided into two portions (dotted line in Fig. 4) which retain the requirements for functional subunits. The hisD alleles are heterogeneous with respect to the quantitative nature of accumulations and secondary accumulations (Table 3), growth characteristics (discussed in text), and rates of spontaneous and induced mutation (Hartman, unpublished; Kirchner, unpublished). Similar variation with regard to growth properties and mutation frequencies is also true of mutants of each of the other gene loci, including genes $F$ and $A$, where crude mapping has been performed through the use of deletion mutants (Table 4; Fig. 3). 


\section{Recombination behaviour in the his region}

There is evident no mode of recombination between complementation units, or between genes, that does not occur within the genes or genic subunits. No special positions within the his region have as yet been found where excessive recombination is particularly localized. The general position within the gene where mutation has occurred also seems unrelated to the general ability of the mutant allele to recombine with other alleles or with markers in other genes. Furthermore, the data illustrate, over the eight genes studied, no discrete limiting size for the portion of the chromosome involved in cell-to-cell transfer during transduction or in the process of integration.

Transduction methods are sensitive enough to detect recombination whenever it occurs (Demerec, 1956b). Tests between single-site mutations in which no recombination is detected are thus interpreted as mutations involving the same site. This interpretation must be qualified by two considerations. (1) Certain combinations involving multisite mutations fail to produce wild-type recombinants where such recombination is predicted on the basis of several other, independent, types of examination (e.g. complementation, accumulations). (2) It is possible that so-called single-site mutations do not all truly involve unit chemical elements (for example, single bases or base-pairs). If a mutational event can involve several genetic elements in close juxtaposition (example in Hotchkiss \& Evans, 1958), some mutants may contain multiple lesions while others but single alterations. To fit the experimental data, such hypothetical multiple mutational events must occur more frequently in exceptionally small genetic regions.

Several multisite mutations have the genetic attributes of true deletions: (a) they produce no wild-type recombinants in reciprocal crosses with a number of closely-linked single-site mutations; $(b)$ in some cases they fail to complement two or more adjacent genes (Hartman et al. 1960); (c) several enzymes are absent from cell-free extracts (Ames et al. 1960); $(d)$ in two cases where a test was possible, we were not able to demonstrate replacement of only a portion of the defect with its wild-type allele (i.e. only complete donortype transductions are recovered). Mutant his-57 exhibits the behaviour expected of a lengthy inversion since it can act as donor to give wild-type recombinants with a number of single-site mutants scattered over several adjacent genes $(E F A H B)$ but is unable to serve as recipient with the production of wild-type recombinants. Presumably, recombinations occur in the latter instance but the recombinant genome still carries the inversion, whereas in the former case similar 'replacements' of short regions of genetic material can lead to viable wild-type recombinants. Either the limiting size and possible uniform composition of transducing fragments (Ozeki, 1959), or concommitant deletion of gene region $C D G$ could explain the failure of his-57 to produce wild-type recombinants when infected with phage grown on wildtype bacteria.

The behaviour of strains his-12 and his-24, and of his-2 and his-211, indicate that different mutations at the same site may differentially affect 
the degree of recombination in nearby regions. Further aberrant results in recombination tests were previously reported (Hartman, 1956; Demerec, Goldman \& Lahr, 1958) and the analysis is extended here (e.g. data in Tables 5 and 6). It is possible that synapsis ('effective pairing'; see Chase \& Doermann, 1958, and papers cited therein) is impaired in regions adjacent to sites of mutation. That multisite mutations are extreme in their recombination behaviour makes effective pairing even more suspect as a contributory factor. On the other hand, the identity in recombination behaviour of strains of diverse origin (LT-2 and LT-7; see Lilleengren, 1948) argues against a profound influence of synapsis on the phenomena observed, unless one makes the unlikely assumption that the two strains are structurally identical in small chromosome regions (i.e. that the genes of the two wildtype strains represent truly identical alleles, rather than isoalleles). While the data suggest that the mutated site affects the amount of recombination which may take place over a region which extends for as many as seven genes distant, this effect is exerted very close to, if not at, the mutated site itself.

The calculations of Demerec $(1956 b)$ indicate that a high frequency of genetic exchanges occurs between host chromosome and transduced material. Supporting this contention is the high degree of recombination observed between mutant his sites which, with regard to their position relative to other markers, are placed extremely close together on the map. For example, some sites within a single his gene are separately integrated $20-30 \%$ of the time (see Clowes, 1958), while their dissociation during recombination from markers several genes away rises little higher than $50 \%$ (see data in Hartman, 1956). The degree of recombination allowed is characteristic of each allele and is exerted when its behaviour is measured in combination with his mutations localized to either side of it on the map. The data thus support the contention of Demerec et al. (1958) who suggested that a mutated site might instigate a genetic switch to replication of the wild-type allele on the paired element. The probability that such an event would occur would remain constant regardless of the strain of origin of the companion element (LT-2 or LT-7), in keeping with the results reported in this paper (see Order of mutant sites within single genes). Further, such effects in two-point tests would be expected to be cumulative, but not strictly additive; this was observed (data in Tables 5 and 6). We propose that there is a very high rate of genetic exchange at the chromosomal level following transduction, and that this high frequency may be occasioned or decreased by structural changes (mutations) of both the multisite- and the single-site types.

\section{Some speculations regarding the linkage of histidine genes}

It is not unusual to find newly isolated enteric bacteria with growth requirements for amino acids, etc. (references in Stokes \& Bayne, 1958). It is logical to assume that the prototrophic and the naturally occurring auxotrophic strains each have selective advantages under special conditions in nature. Furthermore, the histidine mutants of Salmonella appear to be at no selective disadvantage to wild-type in the presence of excess L-histidine; there 
may even be some inhibition of the growth rates of small numbers of wildtype bacteria in populations predominantly mutant (Ryan \& Schneider, 1949; and inhibition of wild-type growth on certain enriched media as mentioned in this paper). Micro-organisms with two independent genetic blocks in a single metabolic pathway are known which have a selective advantage over one of the single mutants involved (Davis, 1952; Mitchell \& Mitchell, 1950). The current report describes completely stable multisite mutations, some of which involve more than one gene (other cases were reviewed by Demerec, 1956 b; Demerec \& Hartman, 1959). Further analysis of histidine mutants (Hartman et al. 1960) indicates that there may be a 'position effect' in the case of some mutations, i.e. some mutations localized in a certain gene may also affect the expression of (an) adjacent gene(s). In a haploid organism where the ensemble of the above conditions exists, preferential linkage of genes relating to single biochemical pathways would appear to require but two additional assumptions: (a) that only a portion of the genome is usually transferred between bacterial cells (evidence for this point of view is summarized in Hartman, 1957; Hartman \& Goodgal, 1959); (b) that these processes of partial genetic transfer are important in the evolution of the organisms in nature. The pattern of linkage is thus considered to be a reflexion of the restricted mechanism of recombination available to enteric bacteria, coupled with the existence of multiple or stable genetic lesions which are often not selected against. It is possible, however, that both phenomena are consequences of some other selective force.

One may envisage the localization of all the histidine genes on, let us say, a single molecule of deoxyribonucleic acid. The relatively low content of deoxyribonucleic acid in P22 phage (Garen \& Zinder, 1955) makes it likely that at least two his genes are on a single molecule. If there are conditions under which the functioning (biochemical expression) of this molecule could be temporarily inactivated without affecting its ability to replicate a true copy, it is obvious that the organism would benefit by having on the molecule only those genes relating to a single crucial pathway. A small metabolite pool, sufficient for only one chromosomal duplication, would be required to counteract the inactivating 'event'. Gram-negative bacteria uniquely contain low concentrations of pool metabolites.

The compact arrangement of deoxyribonucleic acid in the chromosome may require unfolding for it to function with high efficiency (review: Gall, 1958). A gene arrangement which allows the simultaneous exposure of the loci responsible for an entire biosynthetic pathway would thus seem desirable. Such dependence of gene function on chromosomal structure might also be reflected in variegated position effects in higher organisms where heterochromatin (in this interpretation tightly coiled material) is often implicated in retarding the expression of nearby genes.

While possibly accounting for a grouping of genes with related functions, the above assumptions do not explain the gene order within the histidine region of the chromosome. In particular, one might expect the genes controlling the first and last steps of the pathway to be adjacent. Perhaps 
elucidation of the nature of the products of the two terminal loci, $E$ and $G$, discussed above, will throw some light on the problems of gene arrangement in relation to gene function in the his region of the Salmonella chromosome.

We are indebted to Dr M. Demerec (Department of Genetics, Carnegie Institution of Washington, Cold Spring Harbor, Long Island, New York, U.S.A.) for most of the mutant strains used in the present work as well as for stimulating discussion during its course. Many of the mutant strains were isolated by Mr Tadashi Miyake. Strains his-195 to his-199 were obtained through the courtesy of Dr E. Goldschmidt (Medical Bacteriology Division, Camp Detrick, Frederick, Maryland, U.S.A.) and strain his-9, inadvertently lost from our collection, was retrieved from Dr F. Kaudewitz (Max-Planck-Institut für Virusforschung, Tübingen, Germany). Dr B. N. Ames contributed many valuable discussions and generously provided us with his data before publication. We also wish to thank Mrs Zlata Hartman and Miss Grace Bert for competent assistance during some stages of the genetic experimentation. This investigation was supported by a research grant, E1650, from the National Institute of Allergy and Infectious Diseases, Public Health Service, U.S.A.

\section{REFERENCES}

Abderhalden, E. \& Weil, A. (1912). Spaltung des racemischen Histidins in seine optisch aktiven Komponenten. Hoppe-Seyl. Z. 77, 441.

Ames, B. N. (1957). The biosynthesis of histidine: L-histidinol phosphate phosphatase. J. biol. Chem. 226, 583.

Ames, B. N. \& Garry, B. (1959). Co-ordinate repression of the synthesis of four histidine biosynthetic enzymes by histidine. Proc. nat. Acad. Sci., Wash. 45, 1453.

Ames, B. N., Garry, B. \& Herzenberg, L. (1960). The genetic control of the enzymes of histidine biosynthesis in Salmonella typhimurium. J. gen. Microbiol. 22, 369.

Ames, B. N. \& Mrtchell, H. K. (1952). The paper chromatography of imidazoles. J. Amer. chem. Soc. 74, 252.

Ames, B. N. \& Mitchell, H. K. (1955). The biosynthesis of histidine. Imidazoleglycerol phosphate, imidazoleacetol phosphate and histidinol phosphate. J. biol. Chem. 212, 687.

Bratton, A. C. \& Marshall, Jun. E. K. (1939). A new coupling component for sulfanilamide determination. $J$. biol. Chem. 128, 537.

Chase, M. \& Doermann, A. H. (1958). High negative interference over short segments of the genetic structure of bacteriophage T4. Genetics, 43, 332.

Clowes, R. C. (1958). Investigation of the genetics of cysteineless mutants of Salmonella typhimurium by transduction. J. gen. Microbiol. 18, 154.

Davis, B. D. (1952). Aromatic biosynthesis. V. Antagonism between shikimic acid and its precursor, 5-dehydroshikimic acid. J. Bact. 64, 749 .

Demerec, M. $(1956 a)$. Terminology and nomenclature. Genetic Studies with Bacteria. Publ. Carneg. Instn, no. 612, p. 104.

Demerec, M. $(\mathbf{1 9 5 6} b)$. A comparative study of certain gene loci in Salmonella. Cold Spr. Harb. Symp. quant. Biol. 21, 113.

Demerec, M., Goldman. I. \& LAhr, E. L. (1958). Genetic recombination by transduction in Salmonella. Cold Spr. Harb. Symp. quant. Biol. 23, 59.

Demerec, M. \& Hartman, P. E. (1959). Complex loci in microorganisms. Annu. Rev. Microbiol. 13, 377.

Demerec, M., Lahr, E. L., Miyake, T., Goldman, I., Balbinder, E., Banic, S., Hashimoto, K., Glanvilue, E. V. \& Gross, J. D. (1958). Bacterial geneties. Yearb. Carneg. Instn, 57, 390.

Gall, J. G. (1958). Chromosomal differentiation. In The Chemical Basis of Development, ed. W. D. McElroy \& B. Glass, pp. 103-35. Baltimore: The Johns Hopkins Press. 
Garen, A. \& Zinder, N. D. (1955). Radiological evidence for partial genetic homology between bacteriophage and host bacteria. Virology, 1, 347.

Hartman, P. E. (1956). Linked loci in the control of consecutive steps in the primary pathway of histidine synthesis in Salmonella typhimurium. Genetic Studies with Bacteria, Publ. Carneg. Instn, no. 612, p. 35.

Hartman, P. E. (1957). Transduction: A comparative review. In The Chemical Basis of Heredity, ed. W. D. McElroy \& B. Glass, pp. 408-462. Baltimore: The Johns Hopkins Press.

Hartman, P. E. \& Goodgal, S. H. (1959). Bacterial genetics (with particular reference to genetic transfer). Annu. Rev. Microbiol. 13, 465.

Hartman, P. E., Hartman, Z. \& Śrman, D. (1960). Complementation mapping by abortive transduction of histidine-requiring Salmonella mutants. J. gen. Microbiol. 22, 354.

Hershey, A. D. \& Chase, M. (1952). Independent functions of viral protein and nucleic acid in growth of bacteriophage. J. gen. Physiol. 36, 39.

Hotchisiss, R. D. \& Evans, A. H. (1958). Analysis of the complex sulfonamide resistance locus of Pneumococcus. Cold Spr. Harb. Symp. quant. Biol. 23, 85.

Kaudewitz, F., Vielmetter, W. \& Friedrich-Freksa, H. (1958). Mutagene Wirkung des Zerfalles von radioaktivem Phosphor nach Einbau in Zellen von Escherichia coli. Z. Naturf. 13 b, 793.

LEDERBERG, J. (1950). Isolation and characterization of biochemical mutants of bacteria. Meth. Med. Res. 3, 5.

Lederberg, J. (1955). Recombination mechanisms in bacteria. J. cell. comp. Physiol. 45, Suppl. 2, 75.

Lederberg, J. \& Lederberg, E. M. (1952). Replica plating and indirect selection of bacterial mutants. J. Bact. 63, 399.

Lilleengen, K. (1948). Typing Salmonella typhimurium by means of bacteriophage. Acta path. microbiol. scand. Suppl. 77, 68, 75.

MagasanIK, B. (1958). The metabolic regulation of purine interconversions and of histidine biosynthesis. In The Chemical Basis of Development, ed. W. D. McElroy \& B. Glass, p. 485. Baltimore: The Johns Hopkins Press.

Mitchell, M. B. \& Mitchell, H. K. (1950). The selective advantage of an adenineless double mutant over one of the single mutants involved. Proc. nat. Acad. Sci., Wash. 36, 115.

Moyed, H.S. \& Friedman, M. (1959). Interference with feedback control : a mechanism of antimetabolite action. Science, 129, 986.

Moyed, H. S. \& MAgasanik, B. (1957). The role of purines in histidine biosynthesis. J. Amer. chem. Soc. 79, 4812.

Moyed, H. S. \& Magasanik, B. (1958). Biosynthesis of the imidazole ring of histidine: genetic and environmental control. Abstr. Commun. 7th Int. Congr. Microbiol. p. 141.

Nomenclature (1958). Microbial Genetics Bull. 16, 38.

Ozekr, H. (1959). Chromosome fragments participating in transduction in Salmonella typhimurium. Genetics, 44, 457 .

Ryan, F. J. \& Schneider, L. K. (1949). The consequences of mutation during the growth of biochemical mutants of Escherichia coli. IV. The mechanism of inhibition of histidine-independent bacteria by histidineless bacteria. J. Bact. 58, 201.

Stokes, J. L. \& BAYNe, H. G. (1958). Growth-factor-dependent strains of Salmonellae. J. Bact. 76, 417.

Vogel, H. J. \& Bonner, D. M. (1956). Acetylornithinase of Escherichia coli: partial purification and some properties. J. biol. Chem. 218, 97.

Vogel, H. J., Davis, B. D. \& Mingroli, E. S. (1951). L-Histidinol, a precursor of L-histidine in Escherichia coli. J. Amer. chem. Soc. 73, 1897. 